\title{
Invasion moving boundary problem for a biofilm reactor model
}

\author{
B. D’ACUNTO, L. FRUNZO, V. LUONGO and M. R. MATTEI \\ Department of Mathematics and Applications, University of Naples “Federico II", Complesso Monte Sant'Angelo, \\ 80124 Naples, Italy \\ emails:dacunto@unina.it,luigi.frunzo@unina.it,vincenzo.luongo@unina.it,mariarosaria.mattei@unina.it
}

(Received 1 August 2017; revised 7 March 2018; accepted 8 March 2018; first published online 5 April 2018)

\begin{abstract}
The work presents the analysis of the free boundary value problem related to the onedimensional invasion model of new species in biofilm reactors. In the framework of continuum approach to mathematical modelling of biofilm growth, the problem consists of a system of non-linear hyperbolic partial differential equations governing the microbial species growth and a system of semi-linear elliptic partial differential equations describing the substrate trends. The model is completed with a system of elliptic partial differential equations governing the diffusion and reaction of planktonic cells, which are able to switch their mode of growth from planktonic to sessile when specific environmental conditions are found. Two systems of non-linear differential equations for the substrate and planktonic cells mass balance within the bulk liquid are also considered. The free boundary evolution is governed by a differential equation that accounts for detachment. The qualitative analysis is performed and a uniqueness and existence result is presented. Furthermore, two special models of biological and engineering interest are discussed numerically. The invasion of Anammox bacteria in a constituted biofilm inhabiting the deammonification units of the wastewater treatment plants is simulated. Numerical simulations are run to evaluate the influence of the colonization process on biofilm structure and activity.
\end{abstract}

Key words: Invasion model, biofilm reactor, hyperbolic free boundary value problem, numerical simulations, Anammox process.

\section{Introduction}

The term biofilm is used nowadays to indicate the prevailing form of microbial lifestyle, which consists of complex dynamic microbial structures composed of various prokaryotic cells and other microorganisms, forming on solid or liquid surfaces and encased in a selfproduced protective matrix of extracellular polymeric substances. The roles biofilms exert on both natural and human environments are disparate: they have proven detrimental to human health or undesirable in the open water environment, but they can also be used beneficially in resource recovery systems, as well as water treatment [1]. With specific reference to the last point, biofilm reactors represent the primary means to harness the usefulness of biofilms for wastewater treatment by means of the synergistic interactions and biochemical transformations characterizing these microbial communities [2]. The biofilm structure results from the interplay of different interactions, such as mass transfer, 
conversion rates and detachment forces. The main biofilm expansion is due to bacterial growth and to extracellular polymer production. The soluble substrates necessary for bacterial growth are dissolved in the liquid flow and reach the cells first by passing through a boundary layer, characterized by a negligible flow over the biofilm/liquid interface, and then through the biofilm matrix. The external fluid flow regulates biofilm growth by establishing the concentration of substrates and products at the solid-liquid interface and shearing the biofilm surface. Other biological phenomena are found to play significant roles in the establishment of mixed species biofilms, i.e. dispersal, bacteriophage and quorum sensing [3].

Among these phenomena, there is a growing interest in the study of microbial invasion and colonization of pre-existing biofilms as these processes can determine biofilm landscape and contribute to rapid alterations in biofilm populations. Recent advances in microbial ecology have identified motility as one of the main mediators of such processes. Indeed, motile bacteria with high kinetic energy and acting as invaders can lead to the dissolution of heterologous biofilms and repopulation of the matrix [4]. Once a motile bacteria, supplied by the liquid phase or the biofilm itself (as a consequence of dispersal phenomenon), has successfully infiltrated the biofilm matrix, it can invade a resident community and establish where the environmental conditions are optimal for its growth. An accurate modelling of such a system has to take all of these factors into account.

Over the last decades, numerous approaches have been proposed to model biofilm development and structure. Two main categories can be distinguished, namely the continuum [5-7] and discrete models [8,9], based on biomass representation (refer to [10] for a recent survey). The one-dimensional multispecies biofilm model proposed by Wanner and Gujer [11] is an essentially hyperbolic free boundary value problem, and it has been widely used for numerical simulations of wastewater treatment bioreactors. However, in a recent contribution [12], it was shown through an exclusion principle that this model leads to restrictions on ecological structure and highlighted the importance of including downward microbial mobility for ecological considerations. This was accomplished in [13] by adding diffusion terms to the equations for the material volume fractions and thus converting the model from hyperbolic to parabolic. In a recent contribution [14], the authors have introduced a multispecies biofilm model which explicitly takes into account the invasion phenomenon pursued by planktonic cells. The core of the model lies on the introduction of new state variables that represent the concentrations of planktonic colonizing cells within the biofilm. These cells are supposed to be characterized by a diffusive movement within the biofilm and be able to give up the ability to move in order to settle down in specific environmental niches.

In this work, we introduce the free boundary value problem for the invasion phenomenon in biofilm reactors which takes into account the dynamics of the bulk liquid phase in terms of both substrates and planktonic cells. The mathematical problem consists of a system of hyperbolic partial differential equations governing the biofilm growth, a system of elliptic partial differential equations for substrate dynamics within the biofilm and a system of elliptic partial differential equations regulating the diffusion and reaction of planktonic cells. Mass balance equations for the dissolved substrates and planktonic cells within the bulk liquid phase of the biofilm reactor have been taken into account 
as well. The free boundary evolution is governed by a non-linear ordinary differential equation. It is noteworthy to cite the work of [15], where the interactions between the two modes of growth, the suspended and the sessile, have been investigated within a biofilm reactor devoted to wastetwater treatment. The exchange between the two phenotypic states has been considered through the attachment and detachment fluxes, but a downward mobility has not been taken into account.

The qualitative analysis of such a complex system is not an easy task as outlined in [15]. Beyond the contribution given by the authors to this topic, only a few qualitative mathematical results can be found in the literature for the Wanner-Gujer model [16,17]. Due to the high non-linearity of the problem, the fixed point theorem seems the natural tool to be used for the existence and uniqueness of the solutions. However, we are considering a moving boundary problem where the domain is not fixed. To overcome this issue, we follow the methodology used in [18] for the analysis of the biofilm reactor model and in $[14,19]$ for the modelling of the planktonic cells dynamics both within the biofilm and the bulk phase. In particular, we use the method of characteristics to convert the differential problem to an integral one where the unknown functions are defined on a fixed domain and the existence and uniqueness of the solutions are proved in the class of continuous functions.

In addition, the work is completed with some numerical applications related to a real engineering/biological case that examines the invasion of specific microbial species in a constituted biofilm. More precisely, the case study reproduces the invasion of Anammox bacteria within a multispecies biofilm devoted to the concurrent oxidization of ammonium nitrogen and organic carbon occurring in the biological units of the wastewater treatment plants. Traditionally, ammonium oxidation leads to the formation of residual nitrogen compounds that need to be further removed by means of other treatment phases. The establishment of a biofilm community constituted by Anammox bacteria and Aerobic ammonium oxidizers may lead instead to the complete conversion of ammonium nitrogen to nitrogen gas within a single treatment unit. The establishment of this syntrophy is catalysed by the formation of an anoxic zone where the Anammox bacteria can effectively proliferate. The invasion model has been adopted to illustrate the trends related to the establishment of such a multispecies community and to assess the effect of specific operational conditions on the biofilm colonization by Anammox bacteria. For all the cases analysed, real data from existing literature is used to feed numerical simulations, which produce results in nice agreement with experimental findings.

The paper is organized as follows. In Section 2, the invasion moving boundary problem for a biofilm reactor model is introduced and assumptions, boundary and initial conditions are discussed. Section 3 introduces the Volterra integral equations. In Section 4, a uniqueness and existence result is presented. Section 5 describes the experimental case to which the model is applied and presents the numerical results. Finally, in Section 6, we present the conclusions and the future recommendations of the work.

\section{Invasion boundary problem for biofilm reactors}

We analyse the free boundary value problem related to the invasion problem in biofilm reactors. In this model, we consider the biofilm as constituted by various particulate 
components (bacteria, extracellular polymeric substances, etc.) growing in a liquid environment along with planktonic cells belonging to various microbial species that are able to move within the biofilm and the bulk liquid as well. The biofilm expansion depends on growth limiting nutrients which are dissolved in the liquid region or produced within the biofilm itself. The planktonic cells can diffuse and invade from the bulk liquid to the biofilm and switch their mode of growth from suspended to sessile when appropriate environmental conditions are found.

The model is formulated for the variables concentration of microbial species in sessile form $X_{i}$, the concentration of planktonic invading cells $\Psi_{i}$, the concentration of dissolved substrates $S_{j}$, all of which are expressed as functions of time $t$ and the spatial coordinate $z$. The substratum is assumed to be placed at $z=0$. The one-dimensional form of the model writes:

$$
\frac{\partial X_{i}}{\partial t}+\frac{\partial}{\partial z}\left(u X_{i}\right)=\rho_{i} r_{M, i}(z, t, \mathbf{X}, \mathbf{S})+\rho_{i} r_{i}(z, t, \mathbf{S}, \boldsymbol{\Psi}), i=1, \ldots, n,
$$

where $\rho_{i}$ denotes the constant density of species $i, u(z, t)$ the biomass velocity at which the microbial mass is displaced with respect to the film support, and $\mathbf{X}=\left(\mathbf{X}_{\mathbf{1}}, \ldots, \mathbf{X}_{\mathbf{n}}\right)$, $\mathbf{S}=\left(\mathbf{S}_{1}, \ldots, \mathbf{S}_{\mathbf{m}}\right), \boldsymbol{\Psi}=\left(\Psi_{1}, \ldots, \Psi_{n}\right)$. The reaction terms $r_{M, i}$ describe the growth of sessile cells, which is controlled by the local availability of nutrients and usually modelled as standard Monod kinetics, and natural cell death. In most biological processes, the function $r_{M, i}$ depends on $z, t$ only trough the functions $\mathbf{X}$ and $\mathbf{S}$. The explicit dependence has been considered mainly for mathematical generality. The variable $t$ is positive and $0 \leqslant z \leqslant L(t)$, where $L(t)$ denotes the biofilm thickness at time $t$. Equation (2.1) without the term $r_{i}$ was first derived in [11] by mass balance principle. The initial conditions for (2.1) are provided by the initial concentrations $\varphi_{i}(z)$ of biofilm particulate components:

$$
X_{i}(z, 0)=\varphi_{i}(z), i=1, \ldots, n, 0 \leqslant z \leqslant L(0) .
$$

The initial concentrations of the invading microbial species are set to zero. The equation in the form (2.1) was presented in [14]. The terms $r_{i}$ represent the growth rates of the microbial species $X_{i}$ due to the invasion process which induces the switch of planktonic cells to a sessile mode of growth. This phenotypic alteration is catalysed by the formation within the biofilm matrix of specific environmental niches. The explicit dependence on $z, t$ has been introduced only for mathematical generality.

Similarly to traditional continuum models of biofilm growth, equation (2.1) can be rewritten in terms of volume fractions $f_{i}=X_{i} / \rho_{i}$, which indicate the fraction of space at a particular location that is occupied by species $i$,

$$
\frac{\partial f_{i}}{\partial t}+\frac{\partial}{\partial z}\left(u f_{i}\right)=r_{M, i}+r_{i}
$$

It is assumed that the sum of the biomass volume fractions is equal to one at each location and time, $\sum_{i=1}^{n} f_{i}=1$. 
From the equations above, it follows immediately that the function $u(z, t)$ satisfies the following problem:

$$
\frac{\partial u}{\partial z}=\sum_{i=1}^{n}\left(r_{M, i}(z, t, \mathbf{X}, \mathbf{S})+r_{i}(z, t, \mathbf{S}, \boldsymbol{\Psi})\right), 0<z \leqslant L(t), u(0, t)=0,
$$

where the boundary condition $u(0, t)=0$ comes from a no flux condition on substratum.

The function $L(t)$ is solution of the following problem:

$$
\dot{L}(t)=u(L(t), t)-\sigma_{d}(L(t)), L(0)=L_{0}
$$

Therefore, it is apparent that the evolution of the free boundary depends on the displacement velocity of microbial biomass $u$ and detachment flux $\sigma_{d}$ as well. Equation (2.4) comes from the global mass conservation principle.

The substrate diffusion within the biofilm is governed by the following reaction-diffusion equations:

$$
\frac{\partial S_{j}}{\partial t}-\frac{\partial}{\partial z}\left(D_{j} \frac{\partial S_{j}}{\partial z}\right)=r_{S, j}(z, t, \mathbf{X}, \mathbf{S}), j=1, \ldots, m, 0<z<L(t),
$$

where the terms $r_{S, j}$ represent the substrate production or consumption rates due to microbial metabolism and $D_{j}$ denotes the diffusion coefficient of substrate $j$ within the biofilm. As to the boundary conditions, it is assumed that

$$
\frac{\partial S_{j}}{\partial z}(0, t)=0, h \frac{D_{j}}{D_{j}^{*}} \frac{\partial S_{j}}{\partial z}(L(t), t)+S_{j}(L(t), t)=S_{j}^{*}(t), j=1, \ldots, m .
$$

The first condition is a no-flux boundary condition on the substratum placed at $z=0$. The second condition derives from the following reasonings. According to [20], we assume that at a certain distance from the substratum $H(t)=L(t)+h$, with $h$ being a given positive constant, the substrate concentration $S_{j}(H(t), t)$ is the same as the bulk liquid concentration denoted by $S_{j}^{*}(t)$. This dissolved substrate diffuses from the bulk liquid to the biofilm $0 \leqslant z \leqslant L(t)$, where it is consumed according to equation (2.5). No biochemical reactions are supposed to occur for $L(t) \leqslant z \leqslant H(t)$ which leads to consider homogeneous parabolic equations for $S_{j}(z, t)$. Solving at steady-state leads to (2.6), where $D_{j}^{*}$ represents the diffusion coefficient of substrate $j$ within the bulk liquid. Note that condition (2.6) reduces to $S_{j}(L(t), t)=S_{j}^{*}(t)$ for $h=0$.

The functions $S_{j}^{*}(t)$ are governed by the following initial value problem for ordinary differential equations:

$$
V \dot{S}_{j}^{*}=-A D_{j} \frac{\partial S_{j}}{\partial z}(L(t), t)+Q\left(S_{j}^{\text {in }}-S_{j}^{*}(t)\right), j=1, \ldots, m, S_{j}^{*}(0)=S_{j}^{\text {in }} .
$$

Equations above are derived from mass balance on the bulk liquid taking into account the inlet and outlet flux from the reactor and the exchange flux between the biofilm and the bulk liquid. The bulk liquid is modelled as a completely mixed compartment of volume $V$, continuously fed and withdrawn at the same flow rate $Q$. $A$ denotes the biofilm surface area. The initial conditions for $S_{j}^{*}$ are the same as the inlet concentrations. 
The movement of planktonic cells within the biofilm matrix has been modelled through the Fick's law of diffusion as we assumed a random character of motility. An experimental explanation of it can be found in [21]. However, the mechanisms which regulate free cells movement within the biofilm matrix are still poorly understood and further experimental work is required to make definitive assignments on the factors that favour/impede mobility. According to [14], we assume that the presence of relatively large channels and pores within the matrix structure allow the planktonic cells to move within the biofilm itself. The diffusion of planktonic cells within the biofilm matrix is governed by the following diffusion-reaction equations:

$$
\frac{\partial \Psi_{i}}{\partial t}-\frac{\partial}{\partial z}\left(D_{M, i} \frac{\partial \Psi_{i}}{\partial z}\right)=r_{\Psi, i}(z, t, \mathbf{S}, \boldsymbol{\Psi}), i=1, \ldots, n, 0<z<L(t),
$$

where the reaction terms $r_{\Psi, i}$ represent loss terms for the invading species and $D_{M, i}$ denotes the diffusion coefficient for the planktonic species $\Psi_{i}$ in the biofilm. Homogeneous Neumann conditions are adopted on the substratum at $z=0$ due to a no-flux condition, and Robin boundary conditions are prescribed on the free boundary $z=L(t)$ :

$$
\frac{\partial \Psi_{i}}{\partial z}(0, t)=0, h \frac{D_{M, i}}{D_{M, i}^{*}} \frac{\partial \Psi_{i}}{\partial z}(L(t), t)+\Psi_{i}(L(t), t)=\psi_{i}^{*}(t), i=1, \ldots, n,
$$

with $D_{M, i}^{*}$ denoting the diffusion coefficient of planktonic species within the bulk liquid. The initial conditions are set to zero if it is assumed that the invasion process starts at $t=0$, but specific functions can be also considered.

The functions $\psi_{i}^{*}(t)$ denote the concentrations of planktonic cells within the bulk liquid and are governed by the following initial value problem for ordinary differential equations:

$$
V \dot{\psi}_{i}^{*}=-\mathrm{AD}_{M, i} \frac{\partial \Psi_{i}}{\partial z}(L(t), t)+Q\left(\psi_{i}^{\text {in }}-\psi_{i}^{*}(t)\right), \psi_{i}^{*}(0)=\psi_{i}^{\text {in }}, i=1, \ldots, n .
$$

Equation (2.10) come from a mass balance within the bulk liquid and account for the inlet and outlet flux to the biofilm reactor and the exchange fluxes to or from the biofilm as well. The initial concentrations of planktonic cells within the bulk liquid are provided by the inlet concentrations $\psi_{i}^{\text {in }}$.

Finally, due to the slow evolution of the system [18], $S_{j}(z, t)$ profiles can be considered to evolve quasi-statically and thus equation (2.5) are rewritten as

$$
-D_{j} \frac{\partial^{2} S_{j}}{\partial z^{2}}=r_{S, j}(z, \mathbf{X}, \mathbf{S}), j=1, \ldots, m, 0<z<L(t),
$$

with boundary conditions (2.6). In addition, same arguments as before lead to replace equation (2.8) with the following:

$$
-D_{M, i} \frac{\partial^{2} \Psi_{i}}{\partial z^{2}}=r_{\Psi, i}(z, \mathbf{S}, \boldsymbol{\Psi}), i=1, \ldots, n, 0<z<L(t),
$$

with boundary conditions (2.9).

In conclusion, the invasion free boundary problem for biofilm reactor is expressed by equations (2.1)-(2.12). In the next section, following $[18,19]$, an equivalent integral 
formulation of the problem will be provided. As it will be apparent at the end of the following section, the integral form of the free boundary problem presents the great advantage that the space variable is defined on a fixed domain, whereas in the differential formulation (2.1)-(2.12), the space variable belongs to the moving domain $0 \leqslant z \leqslant L(t)$.

\section{Volterra integral equations}

The differential problem introduced in the previous section is herein converted to Volterra integral equations by using the method of characteristics. The characteristic-like lines of system (2.1) are defined by

$$
\frac{\partial c}{\partial t}\left(z_{0}, t\right)=u\left(c\left(z_{0}, t\right), t\right), c\left(z_{0}, 0\right)=z_{0}, 0 \leqslant z_{0} \leqslant L_{0}, t>0
$$

Considering (3.1), equation (2.1) is converted to

$$
\begin{aligned}
& \frac{d}{d t} X_{i}\left(c\left(z_{0}, t\right), t\right) \\
& \quad=F_{i}\left(c\left(z_{0}, t\right), t, \mathbf{X}\left(c\left(z_{0}, t\right), t\right), \mathbf{S}\left(c\left(z_{0}, t\right), t\right), \boldsymbol{\Psi}\left(c\left(z_{0}, t\right), t\right)\right), 0 \leqslant z_{0} \leqslant L_{0}, t>0,
\end{aligned}
$$

with

$$
\begin{aligned}
F_{i}= & \rho_{i} r_{M, i}\left(c\left(z_{0}, t\right), t, \mathbf{X}\left(c\left(z_{0}, t\right), t\right), \mathbf{S}\left(c\left(z_{0}, t\right), t\right)\right) \\
& +\rho_{i} r_{i}\left(c\left(z_{0}, t\right), t, \mathbf{S}\left(c\left(z_{0}, t\right), t\right), \boldsymbol{\Psi}\left(c\left(z_{0}, t\right), t\right)\right)-X_{i}\left(c\left(z_{0}, t\right), t\right) \sum_{i=1}^{n}\left(r_{M, i}+r_{i}\right),
\end{aligned}
$$

and initial conditions

$$
X_{i}\left(c\left(z_{0}, 0\right), 0\right)=\varphi_{i}\left(z_{0}\right), \quad 0 \leqslant z_{0} \leqslant L_{0} .
$$

Integrating (3.2) and considering (3.4) yields

$$
\begin{aligned}
X_{i}\left(c\left(z_{0}, t\right), t\right)= & \int_{0}^{t} F_{i}\left(c\left(z_{0}, \tau\right), \tau, \mathbf{X}\left(c\left(z_{0}, \tau\right), \tau\right), \mathbf{S}\left(c\left(z_{0}, \tau\right), \tau\right), \boldsymbol{\Psi}\left(c\left(z_{0}, \tau\right), \tau\right)\right) d \tau \\
& +\varphi_{i}\left(z_{0}\right), \quad i=1, \ldots, n, 0 \leqslant z_{0} \leqslant L_{0}, t>0 .
\end{aligned}
$$

The following integral equation for $c\left(z_{0}, t\right)$ is derived from (3.1) and (2.3):

$$
\begin{aligned}
c\left(z_{0}, t\right)= & z_{0}+\int_{0}^{t} d \tau \int_{0}^{z_{0}} \sum_{i=1}^{n}\left(\left(r_{M, i}\left(c\left(\zeta_{0}, \tau\right), \tau, \mathbf{X}\left(c\left(\zeta_{0}, \tau\right), \tau\right), \mathbf{S}\left(c\left(\zeta_{0}, \tau\right), \tau\right)\right)\right.\right. \\
& \left.+r_{i}\left(c\left(\zeta_{0}, \tau\right), \tau, \mathbf{S}\left(c\left(\zeta_{0}, \tau\right), \tau\right), \boldsymbol{\Psi}\left(c\left(\zeta_{0}, \tau\right), \tau\right)\right)\right) \frac{\partial c}{\partial \zeta_{0}}\left(\zeta_{0}, \tau\right) d \zeta_{0}, 0 \leqslant z_{0} \leqslant L_{0}, t>0 .
\end{aligned}
$$

From (3.6), it follows easily

$$
\begin{aligned}
\frac{\partial c}{\partial z_{0}}\left(z_{0}, t\right)= & 1+\int_{0}^{t} \sum_{i=1}^{n}\left(\left(r_{M, i}\left(c\left(z_{0}, \tau\right), \tau, \mathbf{X}\left(c\left(z_{0}, \tau\right), \tau\right), \mathbf{S}\left(c\left(z_{0}, \tau\right), \tau\right)\right)\right.\right. \\
& \left.+r_{i}\left(c\left(z_{0}, \tau\right), \tau, \mathbf{S}\left(c\left(z_{0}, \tau\right), \tau\right), \boldsymbol{\Psi}\left(c\left(z_{0}, \tau\right), \tau\right)\right)\right) \frac{\partial c}{\partial \zeta_{0}}\left(z_{0}, \tau\right) d \tau, 0 \leqslant z_{0} \leqslant L_{0}, t>0
\end{aligned}
$$


The integral equations for $S_{j}(z, t)$ are obtained by integrating (2.11) and considering the boundary conditions $(2.6)$

$$
\begin{aligned}
S_{j}(z, t)= & S_{j}^{*}(t)+D_{j}^{-1} \int_{0}^{z}(L-z) r_{S, j}(\zeta, \mathbf{X}(\zeta, t), \mathbf{S}(\zeta, t)) d \zeta \\
& +D_{j}^{-1} \int_{z}^{L}(L-\zeta) r_{S, j}(\zeta, \mathbf{X}(\zeta, t), \mathbf{S}(\zeta, t)) d \zeta \\
& +\frac{h}{D_{j}^{*}} \int_{0}^{L} r_{S, j}(\zeta, \mathbf{X}(\zeta, t), \mathbf{S}(\zeta, t)) d \zeta, j=1, \ldots, m, 0<z<L(t), t>0 .
\end{aligned}
$$

Similarly, the following integral equations for $\Psi_{i}$ are obtained:

$$
\begin{aligned}
\Psi_{i}(z, t)= & \psi_{i}^{*}(t)+D_{M, i}^{-1} \int_{0}^{z}(L-z) r_{\psi, i}(\zeta, \mathbf{S}(\zeta, t), \boldsymbol{\Psi}(\zeta, t)) d \zeta \\
& +D_{M, i}^{-1} \int_{z}^{L}(L-\zeta) r_{\psi, i}(\zeta, \mathbf{S}(\zeta, t), \boldsymbol{\Psi}(\zeta, t)) d \zeta \\
& +\frac{h}{D_{M, i}^{*}} \int_{0}^{L} r_{\psi, i}(\zeta, \mathbf{S}(\zeta, t), \boldsymbol{\Psi}(\zeta, t)) d \zeta, i=1, \ldots, n, \quad 0<z<L(t), t>0 .
\end{aligned}
$$

From (3.8), it follows

$$
\frac{\partial S_{j}}{\partial z}(L, t)=-D_{j}^{-1} \int_{0}^{L} r_{S, j}(\zeta, \mathbf{X}(\zeta, t), \mathbf{S}(\zeta, t)) d \zeta
$$

Considering (3.10) in (2.7), the equation for $S_{j}^{*}(t)$ writes

$$
\dot{S}_{j}^{*}(t)=(A / V) \int_{0}^{L} r_{S, j}(\zeta, \mathbf{X}(\zeta, t), \mathbf{S}(\zeta, t)) d \zeta+(Q / V)\left(S_{j}^{\text {in }}-S_{j}^{*}(t)\right) .
$$

Integrating the last equation over time leads to the following integral equation for $S_{j}^{*}(t)$ :

$$
\begin{aligned}
S_{j}^{*}(t)= & \int_{0}^{t} \exp (-Q(t-\tau) / V) d \tau \int_{0}^{L}(A / V) r_{S, j}(\zeta, \mathbf{X}(\zeta, \tau), \mathbf{S}(\zeta, \tau)) d \zeta \\
& +S_{j}^{\text {in }}, \quad j=1, \ldots, m, t>0 .
\end{aligned}
$$

Following the same reasoning, a similar equation is obtained for $\psi_{i}^{*}(t)$

$$
\begin{aligned}
\psi_{i}^{*}(t)= & \int_{0}^{t} \exp (-Q(t-\tau) / V) d \tau \int_{0}^{L}(A / V) r_{\psi, i}(\zeta, \mathbf{S}(\zeta, \tau), \boldsymbol{\Psi}(\zeta, \tau)) d \zeta \\
& +\psi_{i}^{\text {in }}, \quad i=1, \ldots, n, t>0 .
\end{aligned}
$$

The integral equation for $L(t)$ is obtained from (2.4)

$$
L(t)=L_{0}+\int_{0}^{t} u(L(\tau), \tau) d \tau-\int_{0}^{t} \sigma_{d}(L(\tau)) d \tau, t>0
$$


Note that under the hypotheses $\sigma_{d}=0$, equations (3.8), (3.9), (3.11) and (3.12) can be re-written by introducing the change of variable $\zeta=c\left(\zeta_{0}, t\right), \zeta_{0}<L_{0}$ and considering that the free boundary $L(t)$ is the characteristic line $z=c\left(L_{0}, t\right)$. They take the following form:

$$
\begin{gathered}
S_{j}\left(c\left(z_{0}, t\right), t\right)=S_{j}^{*}(t)+D_{j}^{-1} \int_{0}^{z_{0}}\left(c\left(L_{0}, t\right)-c\left(z_{0}, t\right)\right) r_{S, j}\left(c\left(\zeta_{0}, t\right), \mathbf{X}\left(c\left(\zeta_{0}, t\right), t\right), \mathbf{S}\left(c\left(\zeta_{0}, t\right), t\right)\right) \\
\times \frac{\partial c}{\partial \zeta_{0}}\left(\zeta_{0}, t\right) d \zeta_{0}+D_{j}^{-1} \int_{z_{0}}^{L_{0}}\left(c\left(L_{0}, t\right)-c\left(\zeta_{0}, t\right)\right) r_{S, j}\left(c\left(\zeta_{0}, t\right), \mathbf{X}\left(c\left(\zeta_{0}, t\right), t\right), \mathbf{S}\left(c\left(\zeta_{0}, t\right), t\right)\right) \\
\times \frac{\partial c}{\partial \zeta_{0}}\left(\zeta_{0}, t\right) d \zeta_{0}+\frac{h}{D_{j}^{*}} \int_{0}^{L_{0}} r_{S, j}\left(c\left(\zeta_{0}, t\right), \mathbf{X}\left(c\left(\zeta_{0}, t\right), t\right), \mathbf{S}\left(c\left(\zeta_{0}, t\right), t\right)\right) \frac{\partial c}{\partial \zeta_{0}}\left(\zeta_{0}, t\right) d \zeta_{0}, \\
j=1, \ldots, m, \quad 0<z_{0}<L_{0}, t>0,
\end{gathered}
$$

$$
\begin{aligned}
\Psi_{i}\left(c\left(z_{0}, t\right), t\right) & \\
= & \psi_{i}^{*}(t)+D_{M, i}^{-1} \int_{0}^{z_{0}}\left(c\left(L_{0}, t\right)-c\left(z_{0}, t\right)\right) r_{\psi, i}\left(c\left(\zeta_{0}, t\right), \mathbf{S}\left(c\left(\zeta_{0}, t\right), t\right), \boldsymbol{\Psi}\left(c\left(\zeta_{0}, t\right), t\right)\right) \\
& \times \frac{\partial c}{\partial \zeta_{0}}\left(\zeta_{0}, t\right) d \zeta_{0}+D_{M, i}^{-1} \int_{z_{0}}^{L_{0}}\left(c\left(L_{0}, t\right)-c\left(\zeta_{0}, t\right)\right) r_{\psi, i}\left(c\left(\zeta_{0}, t\right), \mathbf{S}\left(c\left(\zeta_{0}, t\right), t\right), \boldsymbol{\Psi}\left(c\left(\zeta_{0}, t\right), t\right)\right) \\
& \times \frac{\partial c}{\partial \zeta_{0}}\left(\zeta_{0}, t\right) d \zeta_{0},+\frac{h}{D_{M, i}^{*}} \int_{0}^{L_{0}} r_{\psi, i}\left(c\left(\zeta_{0}, t\right), \mathbf{S}\left(c\left(\zeta_{0}, t\right), t\right), \boldsymbol{\Psi}\left(c\left(\zeta_{0}, t\right), t\right)\right) \frac{\partial c}{\partial \zeta_{0}}\left(\zeta_{0}, t\right) d \zeta_{0}, \\
i= & 1, \ldots, n, 0<z_{0}<L_{0}, t>0, \\
S_{j}^{*}(t)= & S_{j}^{\text {in }}+\int_{0}^{t} \exp (-Q(t-\tau) / V) d \tau \int_{0}^{L_{0}}(A / V) r_{S, j}\left(c\left(\zeta_{0}, t\right), \mathbf{X}\left(c\left(\zeta_{0}, t\right), t\right), \mathbf{S}\left(c\left(\zeta_{0}, t\right), t\right)\right) \\
& \times \frac{\partial c}{\partial \zeta_{0}}\left(\zeta_{0}, t\right) d \zeta_{0}, \quad j=1, \ldots, m, t>0 \\
\psi_{i}^{*}(t)= & \psi_{i}^{\text {in }}+\int_{0}^{t} \exp (-Q(t-\tau) / V) d \tau \int_{0}^{L_{0}}(A / V) r_{\psi, i}\left(c\left(\zeta_{0}, t\right), \mathbf{S}\left(c\left(\zeta_{0}, t\right), t\right), \boldsymbol{\Psi}\left(c\left(\zeta_{0}, t\right), t\right)\right) \\
& \times \frac{\partial c}{\partial \zeta_{0}}\left(\zeta_{0}, t\right) d \zeta_{0}, \quad i=1, \ldots, n, t>0
\end{aligned}
$$

\section{Existence and uniqueness theorem}

As outlined at the end of Section 2, the integral equations introduced in Section 3 depend on time and space variable $z_{0}$ defined in the fixed domain $0 \leqslant z \leqslant L_{0}$. This result is essential to prove the existence and uniqueness of solutions. Indeed, following [18, 19], a suitable contractive map can be introduced in the space of continuous functions and the 
fixed point theorem can be applied. By setting

$$
\begin{array}{r}
x_{i}\left(z_{0}, t\right)=X_{i}\left(c\left(z_{0}, t\right), t\right), \quad i=1, \ldots, n, \quad \mathbf{x}=\left(x_{1}, \ldots, x_{n}\right), \\
s_{j}\left(z_{0}, t\right)=S_{j}\left(c\left(z_{0}, t\right), t\right), \quad j=1, \ldots, m, \quad \mathbf{s}=\left(s_{1}, \ldots, s_{m}\right), \\
\psi_{i}\left(z_{0}, t\right)=\Psi_{i}\left(c\left(z_{0}, t\right), t\right), \quad i=1, \ldots, n, \psi=\left(\psi_{1}, \ldots, \psi_{n}\right),
\end{array}
$$

and introducing the vector of unknown variables $\mathbf{x}^{*}=\left(x_{1}^{*}, \ldots, x_{3 n+2+2 m}^{*}\right)$ such that

$$
\begin{aligned}
x_{i}^{*} & =x_{i}, \quad i=1, \ldots, n, x_{n+1}^{*}=c, x_{n+2}^{*}=\partial c / \partial z_{0}, x_{n+2+j}^{*}=S_{j}^{*}, \\
x_{n+2+m+j}^{*} & =s_{j}, \quad j=1, \ldots, m, x_{n+2+2 m+i}^{*}=\psi_{i}^{*}, x_{2 n+2+2 m+i}^{*}=\psi_{i}, i=1, \ldots, n,
\end{aligned}
$$

the integral equations (3.5)-(3.7), (3.14)-(3.17) are converted to the following more compact equations:

$$
\begin{gathered}
x_{i}^{*}\left(z_{0}, t\right)=\varphi_{i}\left(z_{0}\right)+\int_{0}^{t} F_{i}\left(\tau, \mathbf{x}^{*}\left(z_{0}, \tau\right)\right) d \tau, i=1, \ldots, n, 0 \leqslant z_{0} \leqslant L_{0} \\
x_{n+1}^{*}\left(z_{0}, t\right)=z_{0}+\int_{0}^{t} d \tau \int_{0}^{z_{0}} F_{n+1}\left(\tau, \mathbf{x}^{*}\left(\zeta_{0}, \tau\right)\right) d \zeta_{0}, 0 \leqslant z_{0} \leqslant L_{0} \\
x_{n+2}^{*}\left(z_{0}, t\right)=1+\int_{0}^{t} F_{n+2}\left(\tau, \mathbf{x}^{*}\left(z_{0}, \tau\right)\right) d \tau, 0 \leqslant z_{0} \leqslant L_{0} \\
x_{n+2+j}^{*}(t)=S_{j}^{\text {in }}+\int_{0}^{t} d \tau \int_{0}^{L_{0}} F_{n+2+j}\left(t, \tau, \mathbf{x}^{*}\left(\zeta_{0}, t\right)\right) d \zeta_{0}, j=1, \ldots, m \\
x_{n+2+m+j}^{*}\left(z_{0}, t\right)=x_{n+2+j}^{*}(t)+\int_{0}^{z_{0}} F_{n+2+m+j}^{1}\left(\mathbf{x}^{*}\left(\zeta_{0}, t\right)\right) d \zeta_{0}+\int_{z_{0}}^{L_{0}} F_{n+2+m+j}^{2}\left(\mathbf{x}^{*}\left(\zeta_{0}, t\right)\right) d \zeta_{0} \\
+\int_{0}^{L_{0}} F_{n+2+m+j}^{3}\left(\mathbf{x}^{*}\left(\zeta_{0}, t\right)\right) d \zeta_{0}, j=1, \ldots, m, 0<z_{0}<L_{0}, \\
+\int_{0}^{L_{0}} F_{2 n+2+2 m+i}^{3}\left(\mathbf{x}^{*}\left(\zeta_{0}, t\right)\right) d \zeta_{0}, i=1, \ldots, n, 0<z_{0}<L_{0} \\
x_{2 n+2+2 m+i}^{*}\left(z_{0}, t\right)=x_{n+2+2 m+i}^{*}(t)+\int_{0}^{L_{0}} F_{2 n+2+2 m+i}^{2}\left(\mathbf{x}^{*}\left(\zeta_{0}, t\right)\right) d \zeta_{0} \\
x_{n+2+2 m+i}^{*}(t)=\psi_{i}^{\text {in }}+\int_{0}^{L_{0}} d \tau \int_{0}^{L_{0}} F_{n+2+2 m+i}\left(t, \tau, \mathbf{x}^{*}\left(\zeta_{0}, t\right)\right) d \zeta_{0}, i=1, \ldots, n
\end{gathered}
$$

where the functions $F_{h}, h=1, \ldots, 3 n+2+2 m$ denote the integrand functions in equations (3.5)-(3.7), (3.14)-(3.17). The following result can be proved. 


\section{Theorem 1 Assume that}

(i) the functions $x_{h}^{*}\left(z_{0}, t\right)$ are continuous on $I=\left[0, L_{0}\right] \times\left[0, T_{1}\right], L_{0}>0, T_{1}>0, h=$ $1, \ldots, 3 n+2+2 m$

(ii) $\varphi_{i}\left(z_{0}\right)$ are continuous functions on $I=\left[0, L_{0}\right] \times\left[0, T_{1}\right], L_{0}>0, T_{1}>0, i=1, \ldots, n$, and $S_{j}^{i n}, \psi_{i}^{\text {in }}$ are positive constants $j=1, \ldots, m, i=1, \ldots, n$;

(iii) $\left|x_{i}^{*}-\varphi_{i}\right| \leqslant K_{i}, i=1, \ldots, n ;\left|x_{n+1}^{*}-z_{0}\right| \leqslant K_{n+1} ; 1 \leqslant x_{n+2}^{*} \leqslant 1+K_{n+2} ;\left|x_{n+2+j}^{*}-S_{j}^{i n}\right| \leqslant$ $K_{n+2+j}, j=1, \ldots, 2 m ; \quad\left|x_{n+2+2 m+i}^{*}-\psi_{i}^{i n}\right| \leqslant K_{n+2+2 m+i}, \quad i=1, \ldots, 2 n$, where $K_{h}=$ constant $>0$

(iv) $G=\sum_{i=1}^{n}\left(r_{M, i}\left(c\left(z_{0}, t\right), t, \mathbf{x}\left(z_{0}, t\right), \mathbf{s}\left(z_{0}, t\right)\right)+r_{i}\left(c\left(z_{0}, t\right), t, \mathbf{s}\left(z_{0}, t\right), \psi\left(z_{0}, t\right)\right)\right)$ is essentially positive;

(v) $F_{h}$ are bounded and Lipschitz continuous functions with respect to $x_{h}^{*}, h=1, \ldots, 3 n+$ $2+2 m$,

$$
\begin{aligned}
M_{h} & =\max \left|F_{h}\right|, h=1, \ldots, n+2+m, \\
M_{n+2+m+j}^{1} & =\max \left|F_{n+2+m+j}^{1}\right|, M_{n+2+m+j}^{2}=\max \left|F_{n+2+m+j}^{2}\right|, \\
M_{n+2+m+j}^{3} & =\max \left|F_{n+2+m+j}^{3}\right|, \\
M_{n+2+m+j} & =\max \left\{M_{n+2+m+j}^{1}, M_{n+2+m+j}^{2}, M_{n+2+m+j}^{3}\right\}, j=1, \ldots, m, \\
M_{n+2+2 m+i} & =\max \left|F_{n+2+2 m+i}\right|, i=1, \ldots, n, \\
M_{2 n+2+2 m+i}^{1} & =\max \left|F_{2 n+2+2 m+i}^{1}\right|, M_{2 n+2+2 m+i}^{2}=\max \left|F_{2 n+2+2 m+i}^{2}\right|, \\
M_{2 n+2+2 m+i}^{3} & =\max \left|F_{2 n+2+2 m+i}^{3}\right| \\
M_{2 n+2+2 m+i} & =\max \left\{M_{2 n+2+2 m+i}^{1}, M_{2 n+2+2 m+i}^{2}, M_{2 n+2+2 m+i}^{3}\right\}, i=1, \ldots, n,
\end{aligned}
$$

when $\left(z_{0}, t\right) \in\left[0, L_{0}\right] \times\left[0, T_{1}\right]$ and the functions $x_{h}^{*}$ satisfy the assumptions (i)-(iv).

Then, the integral system (4.1)-(4.7) has a unique solution $x_{h}^{*} \in C^{0}\left(\left[0, L_{0}\right] \times[0, T]\right), h=$ $1, \ldots, 3 n+2+2 m$, where

$$
\begin{gathered}
T=\min \left\{T_{1}, \frac{K_{1}}{M_{1}}, \ldots, \frac{K_{n}}{M_{n}}, \frac{K_{n+1}}{L_{0} M_{n+1}}, \frac{K_{n+2}}{M_{n+2}}, \frac{K_{n+3}}{L_{0} M_{n+3}}, \ldots, \frac{K_{n+2+m}}{L_{0} M_{n+2+m}},\right. \\
\left.\frac{K_{n+2 m+3}}{L_{0} M_{n+2 m+3}}, \ldots, \frac{K_{2 n+2 m+2}}{L_{0} M_{2 n+2 m+2}}\right\} .
\end{gathered}
$$

All the calculations are reported in Appendix A as they are a generalization of $[18,19]$ with small modifications. The previous result represents a crucial point in showing the biological consistency of the proposed model. The existence and uniqueness result has been obtained for an arbitrary number of microbial species $n$ and dissolved substrates $m$, with non-linear reaction terms. The uniqueness result provides a solid base for the further numerical calculations. Moreover, we stress that all hypotheses of the theorem are not suggested by mathematical artefacts, but they are mostly qualitative and naturally derived from biological considerations. 


\section{Anammox invasion model}

In the previous sections, we performed the qualitative analysis for the invasion free boundary value problem of a biofilm reactor model. In particular, a result on the existence and uniqueness of solutions was provided. However, it is apparent that when complex biological cases are discussed, only numerical simulations can provide satisfactory predictions. The previous qualitative analysis gives a solid base for calculations. For the numerical solution of the model, we use an extension of the numerical method proposed in [22]. The method of characteristics is used to track biofilm expansion, while a finite difference approximation is adopted for the diffusion reactions. We extended this method to account for the new independent variables $\Psi_{i}$ which are treated in the same manner of $S_{j}$. A first-order approximation is used for the newly introduced variables $\psi_{i}^{*}$ and $S_{j}^{*}$. An original code is implemented in MatLab platform and simulations are run for a set target simulation time $T$ that will be specified later on. The time to compute the values of the unknown variables is in the order of hours to days, depending on the specific target simulation time.

The simulated biofilm system consists of bacterial cells accumulating on a surface surrounded by an aquatic region and reproduces a typical multiculture and multisubstrate process which establishes in the deammonification units of the wastewater treatment plants. The deammonification process consists of the autotrophic nitrogen removal carried out by two microbial groups, the ammonium oxidizing bacteria AOB $\left(X_{1}\right)$ which oxidize ammonium $S_{\mathrm{NH}_{4}}\left(S_{1}\right)$ partially to nitrite $S_{\mathrm{NO}_{2}}\left(S_{2}\right)$ aerobically, and the anaerobic ammonium oxidizing bacteria $\operatorname{AMX}\left(X_{2}\right)$, which subsequently convert the remaining ammonium and the newly formed nitrite into nitrogen gas and nitrate $S_{\mathrm{NO}_{3}}\left(S_{3}\right)$ in trace concentrations. This process is also known as partial nitritation/anammox [23]. In multispecies biofilms, the AOB and AMX compete with other two major microbial groups: the nitrite oxidizing bacteria $\mathrm{NOB}\left(X_{3}\right)$, which oxidize $S_{\mathrm{NO}_{2}}$ to $S_{\mathrm{NO}_{3}}$ in aerobic conditions and compete with AOB for oxygen $S_{\mathrm{O}_{2}}\left(S_{5}\right)$ and AMX for nitrite, and heterotrophic bacteria $\mathrm{HB}\left(X_{4}\right)$. The latter can be further classified in ordinary heterotrophic organisms oxidizing the organic matter and denitrifiers reducing nitrate to nitrite and nitrite to dinitrogen gas by consuming organic carbon $S_{\mathrm{OC}}\left(S_{4}\right)$. HB compete with $\mathrm{AOB}$ and NOB for oxygen and with NOB for nitrite, the limiting substrate of AMX in most instances. The establishment and proliferation of AMX in such constituted biofilms strictly depends on the formation of an anoxic zone in the inner parts of the matrix where NOB cannot grow due to oxygen limitation.

The mathematical model takes into consideration the dynamics of the five microbial species $X_{i}(z, t)$, including inert material $X_{5}$ which derives from microbial decay, and the five reactive components $S_{j}(z, t)$ within the biofilm. The corresponding concentrations in the bulk liquid $S_{j}^{*}(t)$ are taken into account as well. Planktonic cells have been considered for both AMX and HB species as the model is aimed at simulating the invasion of a constituted biofilm by heterotrophic and Anammox bacteria after the establishment of a favourable environmental niche. Two modelling scenarios have been considered: the case of AMX as a single invading species and the case of AMX and HB invasion and establishment in an autotrophic biofilm. Hereafter, they will be referred as Model 1, considered in Section 5.1, and Model 2 that will be discussed in Section 5.2. 


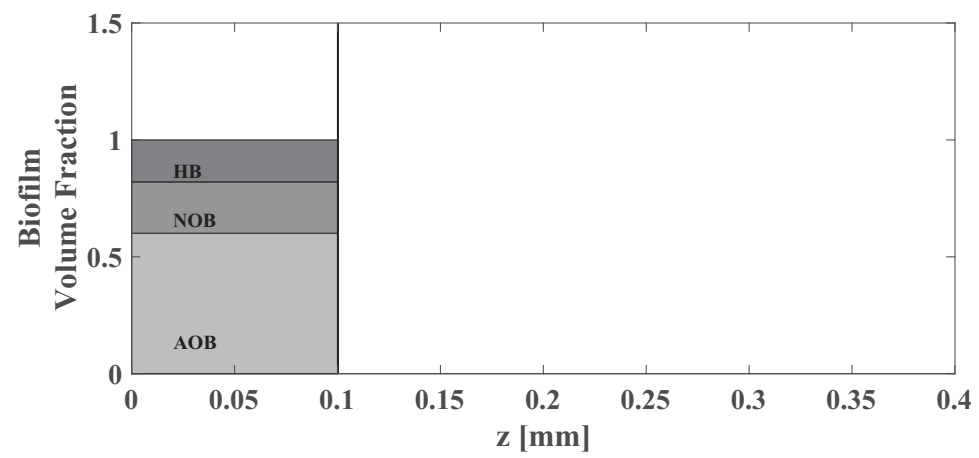

Figure 1. Initial biofilm configuration for Model 1. The substratum is placed at $z=0$.

\subsection{Model 1 - One invading species}

Model 1 considers a single invading species: the anaerobic ammonium oxidizing bacteria AMX $\left(X_{2}\right)$. The mathematical formalization of the problem is provided below. The microbial species dynamics is governed by equation (2.1) rewritten in terms of $f_{i}$ for convenience

$$
\frac{\partial f_{i}}{\partial t}+\frac{\partial}{\partial z}\left(u f_{i}\right)=r_{M, i}(z, t, \mathbf{f}, \mathbf{S})+r_{i}(z, t, \mathbf{S}, \boldsymbol{\Psi}), i=1, \ldots, 5 .
$$

The following initial volume fractions are associated to equation (5.1):

$$
f_{1}(z, 0)=0.6, f_{2}(z, 0)=0.0, f_{3}(z, 0)=0.22, f_{4}(z, 0)=0.18, f_{5}(z, 0)=0.0 .
$$

The biofilm is assumed to be initially constituted only by the species $X_{1}$ (AOB), $X_{3}$ (NOB), $X_{4}(\mathrm{HB})$. The invasion of the species $X_{2}$ (AMX) is simulated. The initial biofilm thickness $L_{0}$ is given by

$$
L_{0}=0.1 \mathrm{~mm} \text {. }
$$

According to the common practice in biofilm modelling [24], the detachment flux $\sigma_{d}(L(t))$ is assumed to be a known function of $L$, in particular,

$$
\sigma_{d}(L(t))=\lambda L^{2}(t)
$$

where $\lambda$ denotes the constant erosion parameter.

A representation of the initial microbial distribution is reported in Figure 1.

The net specific biomass growth rates $r_{M, i}, i=1, \ldots, 4$ are expressed as

$$
r_{M, i}=\left(\mu_{i}(\mathbf{S})-k_{d, i}\right) f_{i}
$$

where the function $\mu_{i}(\mathbf{S})$ denotes the $i$ th biomass specific growth rate and depends on the anabolic reactions performed by the $i$ th microbial species. It is usually formulated as Monod kinetics as detailed below. The term $k_{d, i}$ accounts instead for the forms of biomass loss and energy requirements not associated with growth, including decay, maintenance, endogenous respiration, lysis, predation and death. The net specific growth rates associated 
to $X_{i}, i=1, \ldots, 4$ are the following:

$$
\begin{gathered}
r_{M, 1}=\left(\mu_{1}(\mathbf{S})-k_{d, 1}\right) f_{1}=\left(\mu_{\max , 1} \frac{S_{1}}{K_{1,1}+S_{1}} \frac{S_{5}}{K_{1,5}+S_{5}}-k_{d, 1}\right) f_{1} \\
r_{M, 2}=\left(\mu_{2}(\mathbf{S})-k_{d, 2}\right) f_{2}=\left(\mu_{\max , 2} \frac{K_{2,5}}{K_{2,5}+S_{5}} \frac{S_{1}}{K_{2,1}+S_{1}} \frac{S_{2}}{K_{2,2}+S_{2}}-k_{d, 2}\right) f_{2}, \\
r_{M, 3}=\left(\mu_{3}(\mathbf{S})-k_{d, 3}\right) f_{3}=\left(\mu_{\max , 3} \frac{S_{2}}{K_{3,2}+S_{2}} \frac{S_{5}}{K_{3,5}+S_{5}} \frac{S_{1}}{S_{1}+K_{3,1}}-k_{d, 3}\right) f_{3} \\
=\left(\mu_{\max , 4} \frac{S_{4}}{K_{4,4}+S_{4}} \frac{S_{5}}{K_{4,5}+S_{5}} \frac{S_{1}}{S_{1}+K_{4,1}}+\beta_{1} \mu_{\max , 4} \frac{K_{4,5}}{K_{4,5}+S_{5}} \frac{S_{4}}{K_{4,4}+S_{4}} \frac{S_{3}}{K_{4,3}+S_{3}} \frac{S_{3}}{S_{2}+S_{3}}\right. \\
\left.\times \frac{S_{1}}{S_{1}+K_{4,1}}+\beta_{2} \mu_{\max , 4} \frac{K_{4,5}}{K_{4,5}+S_{5}} \frac{S_{4}}{K_{4,4}+S_{4}} \frac{S_{2}}{K_{4,2}+S_{2}} \frac{S_{2}}{S_{2}+S_{3}} \frac{S_{1}}{S_{1}+K_{4,1}}-k_{d, 4}\right) f_{4}
\end{gathered}
$$

where $\mu_{\text {max }, i}$ denotes the maximum net growth rate for biomass $i, K_{i, j}$ the affinity constant of substrate $j$ for biomass $i, \beta_{1}$ and $\beta_{2}$ the reduction factor for denitrification nitrate to nitrite and nitrite to nitrogen gas, respectively.

The autotrophic performance in the deammonification process relies on the activity of $X_{1}$ (AOB) and $X_{2}(\mathrm{AMX})$ and results in the $S_{1}\left(S_{\mathrm{NH}_{4}}\right)$ conversion to dinitrogen gas via $S_{2}\left(S_{\mathrm{NO}_{2}}\right)$. In aerobic environments, $S_{1}\left(S_{\mathrm{NH}_{4}}\right)$ represents the preferential substrate for $X_{1}$ (AOB) growth (5.4). $X_{2}(\mathrm{AMX})$ proliferate, in turn, on $S_{1}\left(S_{\mathrm{NH}_{4}}\right)$ and $S_{2}\left(S_{\mathrm{NO}_{2}}\right)$ and their metabolic activity is strongly affected by the oxygen concentration, the latter being inhibitory even at low concentrations (5.5). Moreover, they rely on the production of $S_{2}\left(S_{\mathrm{NO}_{2}}\right)$ by $X_{1}$ (AOB), when that substrate is not provided from the bulk liquid. $X_{3}$ (NOB) oxidize $S_{2}\left(S_{\mathrm{NO}_{2}}\right)$ to $S_{3}\left(S_{\mathrm{NO}_{3}}\right)$ under aerobic conditions and thus they compete with $X_{2}$ (AMX) for $S_{2}\left(S_{\mathrm{NO}_{2}}\right)$ (5.6). $X_{4}$ (HB) are considered facultative bacteria: they can aerobically oxidize the organic matter $\left(\mu_{4,1}\right)$ or perform denitrification reactions over $S_{3}\left(S_{\mathrm{NO}_{3}}\right)$ and $S_{2}\left(S_{\mathrm{NO}_{2}}\right)$ ( $\mu_{4,2}$ and $\mu_{4,3}$, respectively). Indeed, in presence of $S_{4}\left(S_{\mathrm{OC}}\right)$, $S_{2}\left(S_{\mathrm{NO}_{2}}\right)$ and $S_{3}\left(S_{\mathrm{NO}_{3}}\right)$ can be contextually consumed by $X_{4}$ (HB) according to equation (5.7). In particular, $S_{3}\left(S_{\mathrm{NO}_{3}}\right)$ and $S_{2}\left(S_{\mathrm{NO}_{2}}\right)$ are reduced to dinitrogen gas in a sequential process which first converts $S_{3}\left(S_{\mathrm{NO}_{3}}\right)$ into $S_{2}\left(S_{\mathrm{NO}_{2}}\right)$, the latter being then reduced to $N_{2}$. In addition, the ratios $S_{3} /\left(S_{2}+S_{3}\right)$ and $S_{2} /\left(S_{2}+S_{3}\right)$ varying between $0 \sim 1$, indicate the percentage of biomass growing on nitrate and/or nitrite, respectively. Inert has been treated as an additional microbial species whose growth rate depends on the decay of all the active species

$$
r_{M, 5}=k_{d, 1} f_{1}+k_{d, 2} f_{2}+k_{d, 3} f_{3}+k_{d, 4} f_{4} .
$$

The specific growth rates $r_{i}$ induced by the switch of the planktonic cells to the sessile mode of growth are defined as

$$
\begin{gathered}
r_{1}=r_{3}=r_{4}=r_{5}=0, \\
r_{2}=k_{c o l, 2} \frac{\Psi_{2}}{k_{\psi, 2}+\Psi_{2}} \frac{K_{2,5}}{K_{2,5}+S_{5}} \frac{S_{1}}{K_{2,1}+S_{1}} \frac{S_{2}}{K_{2,2}+S_{2}} .
\end{gathered}
$$


Note that the growth rate terms $r_{2}$ for $X_{2}$ (AMX) is newly introduced as Monod kinetics and indicate that the transition of bacteria from planktonic state $\Psi_{2}$ into the sessile state $X_{2}$ is controlled by the formation of a specific environmental niche that is strictly connected to the local concentration of dissolved substrates. The presence of planktonic species is fundamental for the occurrence of the invasion process, as better specified in the following lines.

Remark 1 Consider the second equation in (5.1) with $r_{M, 2}$ given by (5.5) and initial condition $f_{2}(z, 0)=0$. If it is supposed that $r_{2}=0$, then the mentioned equation admits the unique solution $f_{2}(z, t)=0$ and the species $X_{2}$ cannot develop.

The diffusion of substrates is governed by

$$
\frac{\partial S_{j}}{\partial t}-D_{j} \frac{\partial^{2} S_{j}}{\partial z^{2}}=r_{S, j}(z, t, \mathbf{X}, \mathbf{S}), j=1, \ldots, 5,
$$

with the following initial boundary conditions:

$$
\begin{gathered}
S_{j}(z, 0)=0, \frac{\partial S_{j}}{\partial z}(0, t)=0, j=1, \ldots, 5, \\
S_{j}(L(t), t)=S_{j}^{*}(t), j=1, \ldots, 4, S_{5}(L(t), t)=\bar{S}_{5}=1.5 \mathrm{mg} O_{2} / L .
\end{gathered}
$$

The last condition simulates a continuous aeration of the biofilm reactor, [15].

The net substrate conversion rates account for both the microbial production and consumption (positive and negative terms, respectively), and can be formulated from the corresponding microbial growth rates through the specific microbial yield $Y_{i}$. They are usually expressed as double-Monod kinetics as presented below.

The ammonium conversion rate $r_{S, 1}$ is expressed as

$$
r_{S, 1}=\left(-\frac{1}{Y_{1}}-i_{N, B}\right) \mu_{1} X_{1}+\left(-\frac{1}{Y_{2}}-i_{N, B}\right) \mu_{2} X_{2}-i_{N, B}\left(\mu_{3} X_{3}+\mu_{4,1} X_{4}+\mu_{4,2} X_{4}+\mu_{4,3} X_{4}\right),
$$

where $Y_{i}$ denotes the yield for biomass $i$ and $i_{N, B}$ is the nitrogen content in biomass. Ammonium can be directly consumed by AOB and AMX (first and second term in (5.14)), and it is usually uptaken by other microbial species for anabolic reactions (third term in (5.14)).

The nitrite and nitrate conversion rates $r_{S, 2}$ and $r_{S, 3}$ can be written as

$$
\begin{gathered}
r_{S, 2}=\frac{1}{Y_{1}} \mu_{1} X_{1}-\left(\frac{1}{Y_{2}}+\frac{1}{1.14}\right) \mu_{2} X_{2}-\frac{1}{Y_{3}} \mu_{3} X_{3}-\left(1-\frac{1}{Y_{4}}\right) \frac{1}{1.14} \mu_{4,2} X_{4}+\left(1-\frac{1}{Y_{4}}\right) \frac{1}{1.72} \mu_{4,3} X_{4} \\
r_{S, 3}=\left(\frac{1}{1.14}\right) \mu_{2} X_{2}+\frac{1}{Y_{3}} \mu_{3} X_{3}+\left(1-\frac{1}{Y_{4}}\right) \frac{1}{1.14} \mu_{4,2} X_{4}
\end{gathered}
$$

In aerobic environments, nitrite $S_{2}$ is produced through the ammonium conversion catalysed by $\mathrm{AOB}$ and it is further oxidized to nitrate $S_{3}$ by NOB (first and third term in (5.15)). Obviously, the latter represents a production rate for nitrate (second term in 
(5.16)). Conversely, in anoxic conditions, AMX bacteria convert nitrite and ammonium in dinitrogen gas (second term in (5.15)), while HB consume both the oxidized nitrogen compounds by reducing nitrate to nitrite (fourth and third term in (5.15) and (5.16), respectively) and by using nitrite as an oxygen source (fifth term in (5.15)).

The organic carbon conversion rate $r_{S, 4}$ is expressed by

$$
r_{S, 4}=-\frac{1}{Y_{4}}\left(\mu_{4,1} X_{4}+\mu_{4,2} X_{4}+\mu_{4,3} X_{4}\right)
$$

and indicates the $S_{4}$ consumption due to $X_{4}$ metabolism in both aerobic and anoxic conditions.

Finally, $r_{S, 5}$ describes the oxygen conversion rate within the multispecies biofilm

$$
r_{S, 5}=\left(1-\frac{3.43}{Y_{1}}\right) \mu_{1} X_{1}+\left(1-\frac{1.14}{Y_{3}}\right) \mu_{3} X_{3}+\left(1-\frac{1}{Y_{4}}\right) \mu_{4,1} X_{4}
$$

where the three terms in (5.18) are the net consumption rates due to $\mathrm{AOB}, \mathrm{NOB}$ and $\mathrm{HB}$ species using oxygen for their metabolisms. The stoichiometric coefficients in equations (5.14)-(5.18) have been calculated from the reactions describing the two-step nitrification process and the oxidation-reduction half reactions related to the denitrification process [25]. They represent (i) 3.43 the amount of oxygen expressed in grams needed for ammonium oxidation to nitrite, (ii) 1.14 the amount of oxygen expressed in grams needed for nitrite oxidation to nitrate, (iii) 1.72 the amount of COD expressed in grams needed to reduce nitrite to dinitrogen gas, (iv) 1.14 the amount of COD expressed in grams necessary to reduce nitrate to nitrite.

The functions $S_{j}^{*}(t)$ are governed by the following initial value problem for ordinary differential equations:

$$
V \dot{S}_{j}^{*}=-\mathrm{AD}_{j} \frac{\partial S_{j}}{\partial z}(L(t), t)+Q\left(S_{j}^{\text {in }}-S_{j}^{*}(t)\right), j=1, \ldots, 4 .
$$

The initial conditions for $S_{j}^{*}$ are the following:

$$
S_{1}^{\text {in }}=1,200 \mathrm{mg} \mathrm{N} / \mathrm{L}, S_{2}^{\text {in }}=S_{3}^{\text {in }}=0, S_{4}^{\text {in }}=120 \mathrm{mg} \mathrm{COD} / \mathrm{L} .
$$

The inlet concentrations are non-zero only for $S_{1}\left(S_{\mathrm{NH}_{4}}\right)$ and $S_{4}\left(S_{\mathrm{OC}}\right)$, reproducing the case of a biofilm reactor fed with wastewater containing both ammonium nitrogen and organic carbon.

The diffusion and reaction of planktonic cells within the biofilm matrix is governed by the following equations:

$$
\frac{\partial \Psi_{i}}{\partial t}-D_{M, i} \frac{\partial^{2} \Psi_{i}}{\partial z^{2}}=r_{\Psi, i}(z, t, \mathbf{S}, \boldsymbol{\Psi}), i=1, \ldots, 5,
$$

where $D_{M, i}$ denotes the diffusivity coefficient. The conversion rates of planktonic cells due to the invasion process are expressed by

$$
r_{\psi, i}=-\frac{1}{Y_{\psi, i}} r_{i}, \quad i=1, \ldots, 5,
$$


with $Y_{\psi, i}$ being the yield of sessile species on planktonic ones. They are assumed proportional to $r_{i}$, i.e. described by the same Monod kinetics [14]. The following initial boundary conditions are associated to equation (5.21):

$$
\Psi_{i}(z, 0)=0, \frac{\partial \Psi_{i}}{\partial z}(0, t)=0, \Psi_{i}(L(t), t)=\psi_{i}^{*}(t), i=1, \ldots, 5
$$

The functions $\psi_{i}^{*}(t)$ satisfy the following initial value problem for ordinary differential equations:

$$
\begin{gathered}
V \dot{\psi}_{i}^{*}=-\mathrm{AD}_{M, i} \frac{\partial \Psi_{i}}{\partial z}(L(t), t)+Q\left(\psi_{i}^{\text {in }}-\psi_{i}^{*}(t)\right), i=1, \ldots, 5, \\
\psi_{1}^{\text {in }}=0, \psi_{2}^{\text {in }}=1.0 \mathrm{mg} \mathrm{COD} / \mathrm{L}, \psi_{3}^{\text {in }}=\psi_{4}^{\text {in }}=\psi_{5}^{\text {in }}=0 .
\end{gathered}
$$

Note that for $i=1$, the equation for $\Psi_{1}$ is homogeneous because of hypothesis (5.9) and equation for $\psi_{1}^{*}$ does not contain the term $\psi_{1}^{\text {in }}$ because of hypothesis (5.25). Therefore, the system of the two equations admits the unique solution $\Psi_{1}(z, t)=0, \psi_{1}^{*}(t)=0$. Same result holds for $\Psi_{3}(z, t)=0, \psi_{3}^{*}(t)=0, \Psi_{4}(z, t)=0, \psi_{4}^{*}(t)=0, \Psi_{5}(z, t)=0, \psi_{5}^{*}(t)=0$.

The biofilm reactor is characterized by the following operational parameters: the flow rate $Q$ is set to $3.15 \mathrm{~L} / \mathrm{d}$, the surface area available for biofilm attachment and proliferation $A$ is equal to $1 \mathrm{~m}^{2}$ and the reactor volume is of $3.15 \mathrm{~L}$, leading to a hydraulic retention time of 1 day. The biofilm mainly grows on the surfaces of suspended carriers, which continuously move due to the aeration system. The reactor is operated in continuous conditions. The biofilm is modelled as a one-dimensional, continuous, homogeneous system growing in a direction perpendicular to the substratum.

The values of the stoichiometric and kinetic parameters used for numerical simulations are adopted from [2] and are reported for convenience in Tables 1 and 2. The values of the erosion parameter $\lambda$ and the diffusion coefficients for the planktonic species $D_{M, i}$ have been reported in Table 2 as well.

The simulation results for the multispecies biofilm performance when the AMX invasion is considered are reported in Figures 2 and 3. After 1 day of simulation time (Figure 2(A)), it is possible to notice that the microbial distribution into the biofilm is still affected by the initial conditions and the colonization phenomenon has not occurred yet. After 5 days of simulation time (Figure 2(B) and (D)), the biofilm experiences oxygen limitation, due to the low concentration maintained within the bulk liquid. As a consequence, the NOB concentration significantly decreases with respect to the initial fraction, with the $\mathrm{AOB}$ and $\mathrm{HB}$ being the two species proliferating the most. The AOB activity is confirmed by the decrease in $S_{\mathrm{NH}_{4}}$ concentration within the bulk liquid with respect to the inlet concentration and a concurrent increase in $S_{\mathrm{NO}_{2}}$ concentration (Figure 2(C) and (D)). Note that the latter is higher than $\mathrm{S}_{\mathrm{NO}_{3}}$ concentration as the metabolic activity of NOB is limited by the low oxygen concentration. The organic carbon is completely depleted within the biofilm and its concentration is lower than $1 \mathrm{mg} / \mathrm{L}$.

Figure 3(A) displays biofilm configuration after 20 days of system operation: nevertheless the concomitant formation of an anoxic zone in the inner part of the biofilm (Figure $3(\mathrm{C})$ ) and a non-zero $\Psi_{2}\left(\psi_{\mathrm{AMX}}\right)$ concentration all over the biofilm (Figures $2(\mathrm{C})$ and (D)-3(C) and (D)), AMX have not yet established in sessile form. This might be due to the very slow growth rate of AMX. The biofilm is dominated by AOB, while 
Table 1. Kinetic and stoichiometric parameters used for numerical simulations

\begin{tabular}{|c|c|c|c|}
\hline Symbol & Definition & Value & Units \\
\hline$Y_{1}$ & $X_{1}$ yield on $S_{1}$ & 0.150 & $\mathrm{~g} \mathrm{COD} / \mathrm{g} \mathrm{N}$ \\
\hline$Y_{2}$ & $X_{2}$ yield on $S_{1}$ & 0.159 & $\mathrm{~g} \mathrm{COD} / \mathrm{g} \mathrm{N}$ \\
\hline$Y_{3}$ & $X_{3}$ yield on $S_{1}$ & 0.041 & $\mathrm{~g} \mathrm{COD} / \mathrm{g} \mathrm{N}$ \\
\hline$Y_{4}$ & $X_{4}$ yield on $S_{4}$ & 0.63 & $\mathrm{~g} \mathrm{COD} / \mathrm{g} \mathrm{COD}$ \\
\hline$\mu_{\max , 1}$ & Maximum growth rate of $X_{1}$ & 2.05 & $\mathrm{~d}^{-1}$ \\
\hline$\mu_{\mathrm{max}, 2}$ & Maximum growth rate of $X_{2}$ & 0.08 & $d^{-1}$ \\
\hline$\mu_{\max , 3}$ & Maximum growth rate of $X_{3}$ & 1.45 & $d^{-1}$ \\
\hline$\mu_{\max , 4}$ & Maximum growth rate of $X_{4}$ & 6.0 & $d^{-1}$ \\
\hline$K_{1,1}$ & $S_{1}$ affinity constant for $X_{1}$ & 2.4 & $\mathrm{mg} \mathrm{N} / \mathrm{L}$ \\
\hline$K_{1,5}$ & $S_{5}$ affinity constant for $X_{1}$ & 0.6 & $\mathrm{mg} \mathrm{O}_{2} / \mathrm{L}$ \\
\hline$K_{2,1}$ & $S_{1}$ affinity constant for $X_{2}$ & 0.07 & $\mathrm{mg} \mathrm{N} / \mathrm{L}$ \\
\hline$K_{2,2}$ & $S_{2}$ affinity constant for $X_{2}$ & 0.05 & $\mathrm{mg} \mathrm{N} / \mathrm{L}$ \\
\hline$K_{2,5}$ & $S_{5}$ inhibiting constant for $X_{2}$ & 0.01 & $\mathrm{mg} \mathrm{O}_{2} / \mathrm{L}$ \\
\hline$K_{3,1}$ & $S_{1}$ affinity constant for $X_{3}$ & 0.1 & $\mathrm{mg} \mathrm{N} / \mathrm{L}$ \\
\hline$K_{3,2}$ & $S_{2}$ affinity constant for $X_{3}$ & 5.5 & $\mathrm{mg} \mathrm{N} / \mathrm{L}$ \\
\hline$K_{3,5}$ & $S_{5}$ affinity constant for $X_{3}$ & 2.2 & $\mathrm{mg} \mathrm{O}_{2} / \mathrm{L}$ \\
\hline$K_{4,4}$ & $S_{4}$ affinity constant for $X_{4}$ & 4.0 & $\mathrm{mg} \mathrm{COD} / \mathrm{L}$ \\
\hline$K_{4,5}$ & $S_{5}$ affinity/inhibiting constant for $X_{4}$ & 0.2 & $\mathrm{mg} \mathrm{O}_{2} / \mathrm{L}$ \\
\hline$K_{4,2}$ & $S_{2}$ affinity constant for $X_{4}$ & 0.5 & $\mathrm{mg} \mathrm{N} / \mathrm{L}$ \\
\hline$K_{4,3}$ & $S_{3}$ affinity constant for $X_{4}$ & 0.5 & $\mathrm{mg} \mathrm{N} / \mathrm{L}$ \\
\hline$K_{4,1}$ & $S_{1}$ affinity constant for $X_{4}$ & 0.1 & $\mathrm{mg} \mathrm{N} / \mathrm{L}$ \\
\hline$k_{d, 1}$ & Decay constant for $X_{1}$ & 0.0068 & $\mathrm{~d}^{-1}$ \\
\hline$k_{d, 2}$ & Decay constant for $X_{2}$ & 0.00026 & $\mathrm{~d}^{-1}$ \\
\hline$k_{d, 3}$ & Decay constant for $X_{3}$ & 0.004 & $d^{-1}$ \\
\hline$k_{d, 4}$ & Decay constant for $X_{4}$ & 0.06 & $d^{-1}$ \\
\hline
\end{tabular}

Table 2. Kinetic and Ssoichiometric parameters used for numerical simulations

\begin{tabular}{lclc}
\hline \hline Symbol & Definition & Value & Units \\
\hline$i_{N, B}$ & $N$ content of biomass & 0.07 & $\mathrm{~g} \mathrm{~N} / \mathrm{g} \mathrm{COD}$ \\
$\beta_{1}$ & Reduction factor for denitrification $\mathrm{NO}_{3}-\mathrm{NO}_{2}$ & 0.8 & - \\
$\beta_{2}$ & Reduction factor for denitrification $\mathrm{NO}_{2}-\mathrm{N}_{2}$ & 0.8 & - \\
$k_{\mathrm{col}, 2}$ & Maximum colonization rate of $\psi_{2}$ & 0.0001 & $\mathrm{~d}^{-1}$ \\
$k_{\mathrm{col}, 4}$ & Maximum colonization rate of $\psi_{4}$ & 0.0001 & $\mathrm{~d}^{-1}$ \\
$Y_{\psi, 2}$ & Yield of $X_{2}$ on $\Psi_{2}$ & 0.001 & - \\
$Y_{\psi, 4}$ & Yield of $X_{4}$ on $\Psi_{4}$ & 0.001 & - \\
$k_{\psi, 2}$ & Kinetic constant for $\Psi_{2}$ & 0.000001 & $\mathrm{mg} \mathrm{COD} / \mathrm{L}$ \\
$k_{\psi, 4}$ & Kinetic constant for $\Psi_{4}$ & 0.000001 & $\mathrm{mg} \mathrm{COD} / \mathrm{L}^{-1}$ \\
$\lambda$ & Erosion parameter & 50 & $\mathrm{~m}^{-1} \mathrm{~d}^{-1}$ \\
$D_{M, 2}$ & Diffusion coefficient for $\Psi_{2}$ & 0.00001 & $\mathrm{~m}^{2} \mathrm{~d}^{-1}$ \\
$D_{M, 4}$ & Diffusion coefficient for $\Psi_{4}$ & 0.00001 & $\mathrm{~m}^{2} \mathrm{~d}^{-1}$ \\
\hline \hline
\end{tabular}


(A)

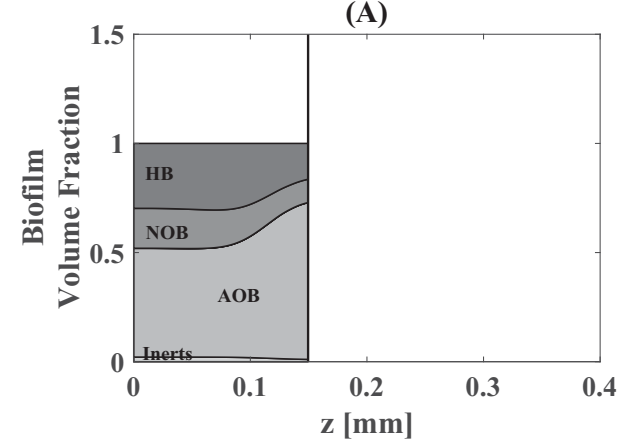

(C)

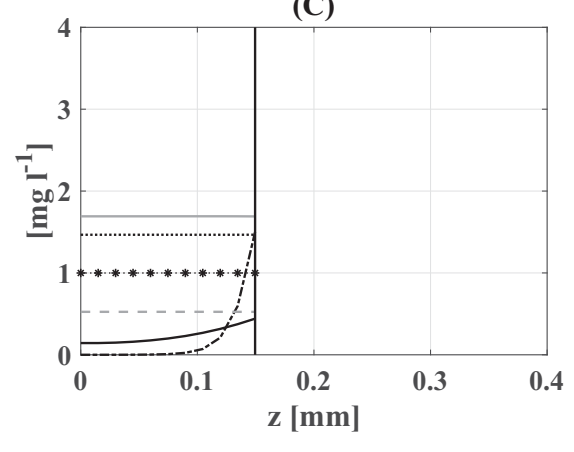

$\cdots \cdots . . . \mathrm{S}_{\mathrm{NH} 4}-\mathrm{S}_{\mathrm{OC}^{-\cdots-. .}} \mathrm{S}_{\mathrm{O} 2}-\mathrm{S}_{\mathrm{NO}^{-\cdots}}-\mathrm{S}_{\mathrm{NO} 3} \cdots * \psi_{\mathrm{AMX}}$
(B)

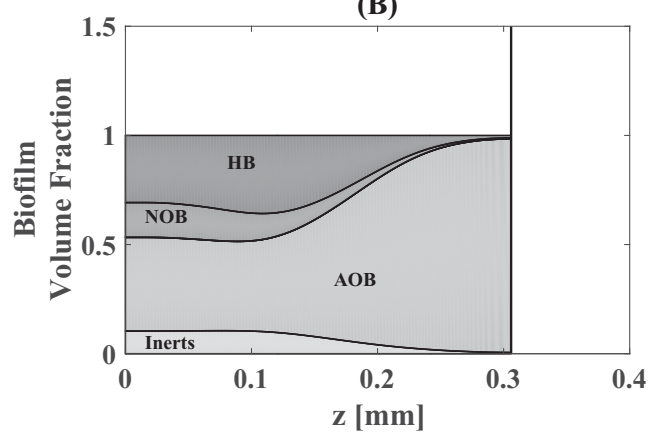

(D)

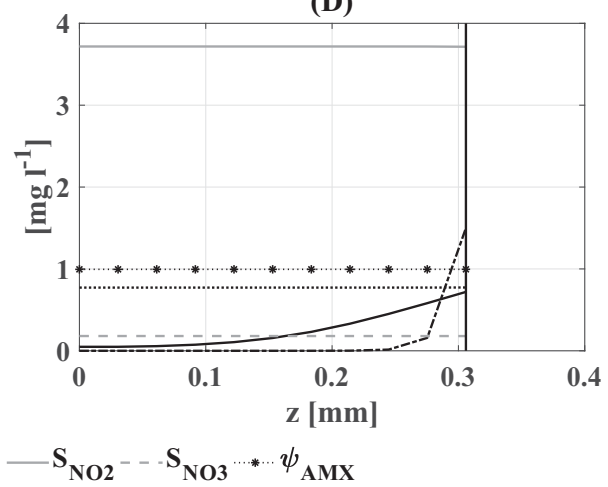

FIGURE 2. Microbial species distribution (A,B) and substrate trends (C,D) within a multispecies biofilm undergoing $\psi_{\mathrm{AMX}}$ colonization after $1(\mathrm{~A}, \mathrm{C})$ and 5(B,D) days simulation time. The substratum is placed at $z=0 . S_{\mathrm{NH}_{4}}, S_{\mathrm{NO}_{2}}$ and $S_{\mathrm{NO}_{3}}$ concentrations are reduced by a factor of $0.002,0.005$ and 0.005 , respectively.

the inert material predominates in the inner layer. Substrate trends assume the following configuration: $S_{\mathrm{NH}_{4}}$ increases in the bulk liquid due to the lower AOB activity in the outer part of the biofilm, where $S_{\mathrm{O}_{2}}$ is totally consumed and its depletion determines the formation of an anoxic zone. Moreover, $S_{\mathrm{NO}_{2}}$ represents the main abundant product, while $S_{\mathrm{NO}_{3}}$ and $S_{\mathrm{OC}}$ are close to zero all over the biofilm (Figure 3(C)). At day 50 (Figure 3(B) and (D)), AMX have colonized the environmental niche that formed at the bottom of the biofilm (Figure 3(B)). AOB still dominate the aerobic zone, while NOB are confined to the internal layers. $S_{\mathrm{NO}_{3}}$ concentration is close to zero as the metabolism of NOB significantly slows down. Note that the availability of $S_{\mathrm{O}_{2}}$ within the biofilm is strictly connected to the relative penetration depth, which when decreased leads to an increasing anoxic zone (Figure 3(D)). Furthermore, AMX grow only where favourable environmental conditions establish despite the biofilm results fully penetrated by the same bacteria in motile/colonizing form $\Psi_{2}$ for all simulation times (Figures 2(B) and (D)-3(B) and (D)).

AMX invasion is significantly influenced by many parameters such as environmental factors (i.e. $\mathrm{pH}$ and temperature) and operational conditions (i.e. dilution rate, organic carbon/nitrogen ratio and aeration pattern). The main goals for the further computational studies are to determine how the invasion phenomenon is affected by the oxygen and 

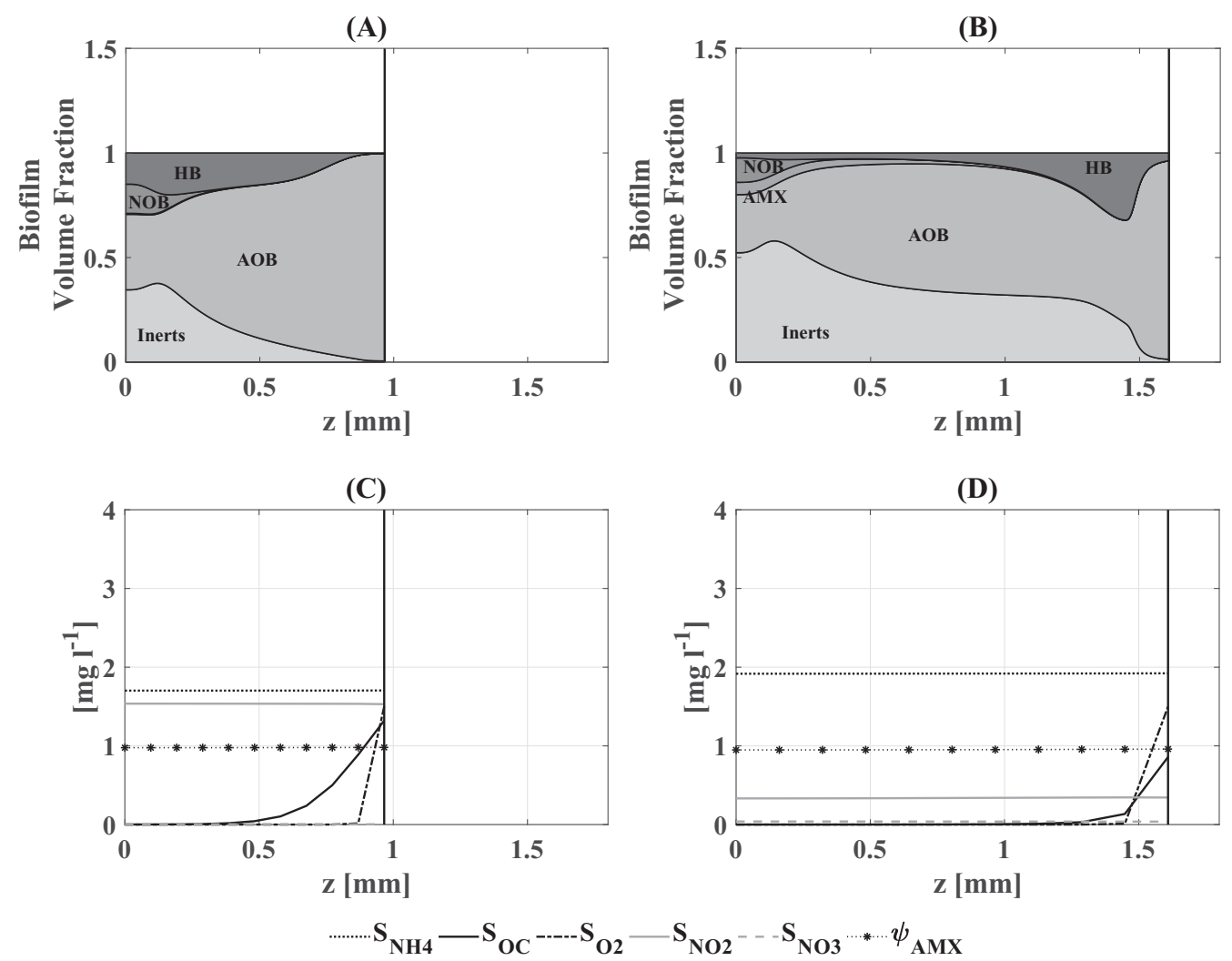

FIGURE 3. Microbial species distribution (A,B) and substrate trends (C,D) within a multispecies biofilm undergoing $\Psi_{2}$ colonization after $20(\mathrm{~A}, \mathrm{C})$ and $50(\mathrm{~B}, \mathrm{D})$ days simulation time. The substratum is placed at $z=0 . S_{\mathrm{NH}_{4}}, S_{\mathrm{NO}_{2}}$ and $S_{\mathrm{NO}_{3}}$ concentrations are reduced by a factor of 0.002 , 0.005 and 0.005 , respectively.

organic carbon availability. For this reason, we vary the concentration of oxygen in the bulk liquid $\bar{S}_{5}$ and the organic carbon concentration in the inlet $S_{4}^{\text {in }}$ in the range [0.5-6] and [120-750], respectively. In the following, we will refer to Sections 5.1.1 and 5.1.2 for the applications with the variable oxygen and organic carbon. We assumed the initial condition reported in Figure 1 for all the simulation studies.

\subsubsection{Model 1 - Assessment A - Effect of oxygen concentration}

Model outcomes for the simulation studies with variable oxygen are summarized in Figures 4 and 5 in terms of biomass distribution and substrate concentrations within the bulk liquid. Four different oxygen levels $\left(0.5-1.5-3-6 \mathrm{mg} \mathrm{O}_{2} / \mathrm{L}\right)$ have been tested and the simulations have been run for a target time of 50 days. AMX are strictly inhibited by the oxygen concentration and, as expected, their total volume fraction is found to slightly increase when varying the oxygen level from 6 to 0.5 . The optimal condition for AMX establishment and proliferation in terms of mass within the biofilm occurs at $3 \mathrm{mg} \mathrm{O} / \mathrm{L}$, although the relative total biofilm fraction is lower with respect to $0.5 \mathrm{mg} \mathrm{O}_{2} / \mathrm{L}$. Of 


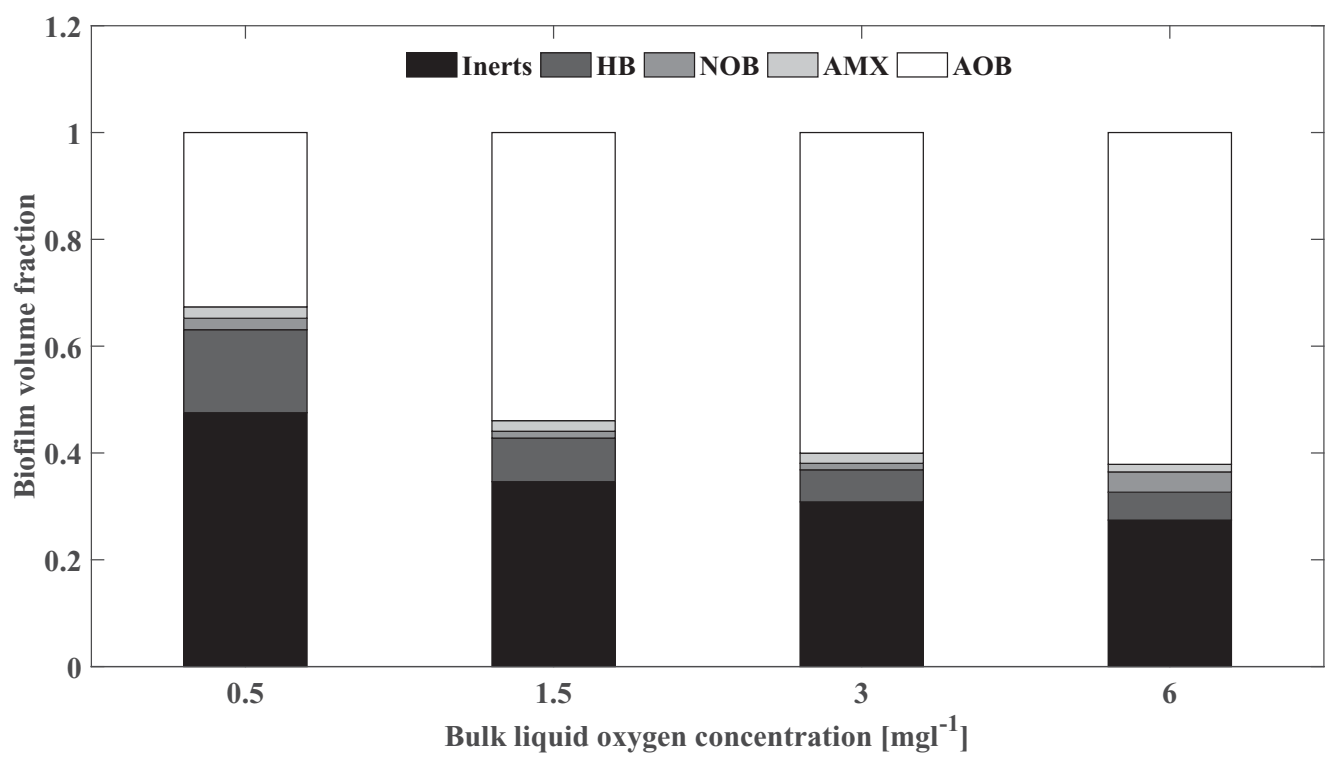

FIGURE 4. Total biofilm volume fractions at different bulk liquid oxygen concentrations after 50 days simulation time.

course, the NOB fraction is higher when the oxygen concentration is equal to $6 \mathrm{mg} \mathrm{O}_{2} / \mathrm{L}$. Regarding nitrogen removal, it is possible to note that the $S_{\mathrm{NH}_{4}}$ concentration progressively decreases and consequently $S_{\mathrm{NO}_{2}}$ increases going from 0.5 to $6 \mathrm{mg} \mathrm{O} / \mathrm{L}$ (Figure 5). These substrates show fully penetrated profiles (data not shown), and consequently the AMX can grow for all the cases with their maximum specific growth rate, but only where anoxic conditions are established.

\subsubsection{Model 1 - Assessment B - Effect of inlet organic carbon concentration}

The second simulation studies investigated the effect of increasing carbon/nitrogen ratios on AMX performances. The oxygen concentration within the bulk liquid has been fixed to $3 \mathrm{mg} \mathrm{O}_{2} / \mathrm{L}$. As shown in Figures 6 and 7, four different concentrations of the inlet organic carbon $S_{4}^{\text {in }}$ have been tested $(120-250-500-750 \mathrm{mg}$ COD/L) and all the simulations have been run for 50 days. Figure 6 shows that the AOB volume fraction is prevalent when low organic carbon is available for $\mathrm{HB}$, which compete for oxygen with all the other aerobic species in the external part of the biofilm. AMX invasion and proliferation (in terms of mass) is favoured at $S_{4}^{\text {in }}=500 \mathrm{mg} \mathrm{COD} / \mathrm{L}$, since the NOB significantly decrease when increasing the inlet organic carbon concentration. The highest carbon content leads to the highest inerts volume fraction as $\mathrm{HB}$ are strongly predominant and out-compete all the other species.

According to the volume fraction distribution, total nitrogen removal is higher when both AMX and AOB can easily perform their metabolisms, while NOB activity is inhibited by HB. This particular condition is more evident when the inlet carbon concentration is $250 \mathrm{mg} \mathrm{COD} / \mathrm{L}$ and a higher amount of dissolved oxygen is utilized by HB. Ammonium removal is not significant when both $\mathrm{AMX}$ and $\mathrm{AOB}$ are not prevalent within the biofilm 


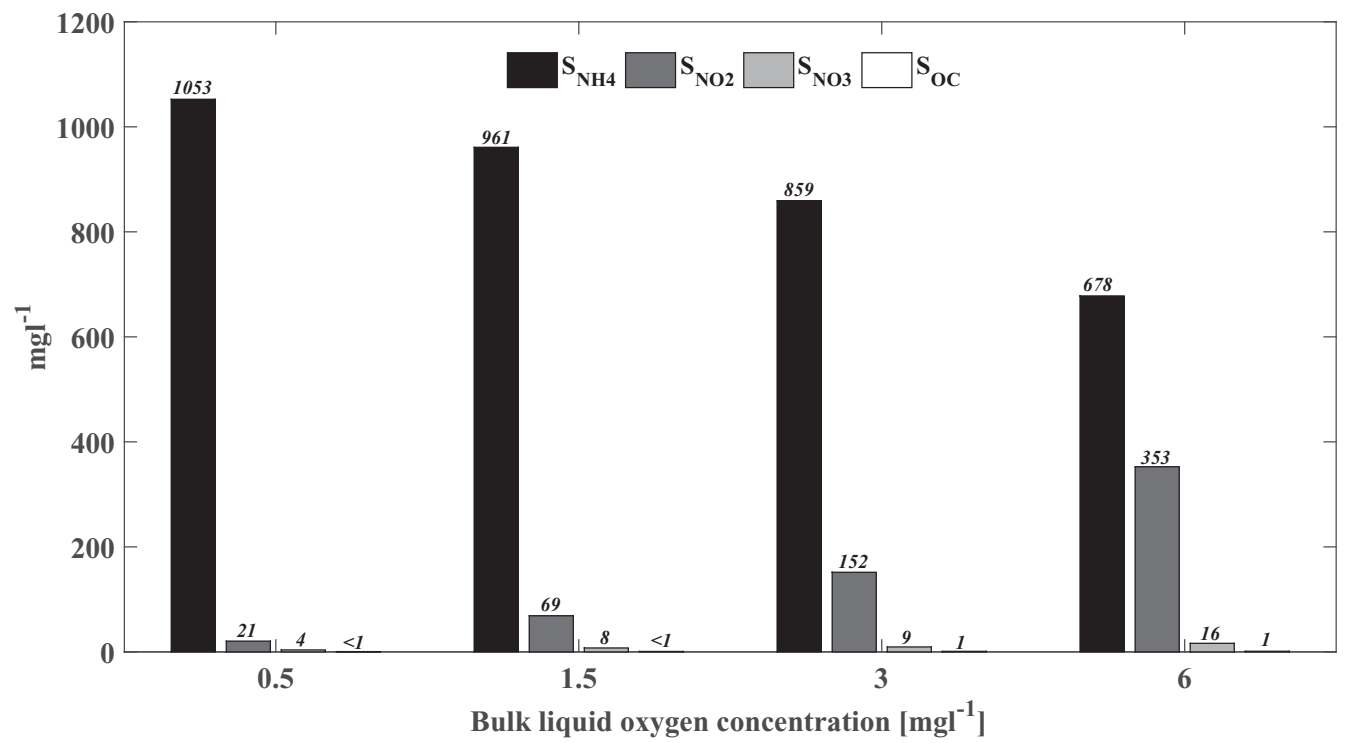

FIGURE 5. Substrate concentrations within the bulk liquid at different bulk liquid oxygen concentrations after 50 days simulation time. The values reported have been rounded to the nearest integer.

and organic carbon removal starts to be incomplete when increasing $S_{4}^{\text {in }}$ concentration to $750 \mathrm{mg} \mathrm{COD} / \mathrm{L}$.

\subsection{Model 2 - Two invading species}

In this section, the model was applied to the case of two species invasion, HB and AMX, respectively. The microbial species growth is governed by equation (5.1) with the following initial volume fractions:

$$
f_{1}(z, 0)=0.7, f_{2}(z, 0)=0, f_{3}(z, 0)=0.3, f_{4}(z, 0)=0, f_{5}(z, 0)=0 .
$$

Only the species $X_{1}(\mathrm{AOB})$ and $X_{3}(\mathrm{NOB})$ are supposed to inhabit the biofilm at $t=0$. The invasion of the species $X_{2}(\mathrm{AMX})$ and $X_{4}(\mathrm{HB})$ is simulated. The initial biofilm thickness $L_{0}$ is given by (5.3). A representation of the initial microbial distribution is reported in Figure 8.

The biomass growth rates $r_{M, i}$ are the same as Model 1, formulas (5.4)-(5.8). The specific growth rates $r_{i}$ induced by the switch of the planktonic cells to the sessile mode of growth are defined as

$$
\begin{gathered}
r_{1}=r_{3}=r_{5}=0, \\
r_{2}=k_{\mathrm{col}, 2} \frac{\Psi_{2}}{k_{\psi, 2}+\Psi_{2}} \frac{K_{2,5}}{K_{2,5}+S_{5}} \frac{S_{1}}{K_{2,1}+S_{1}} \frac{S_{2}}{K_{2,2}+S_{2}},
\end{gathered}
$$




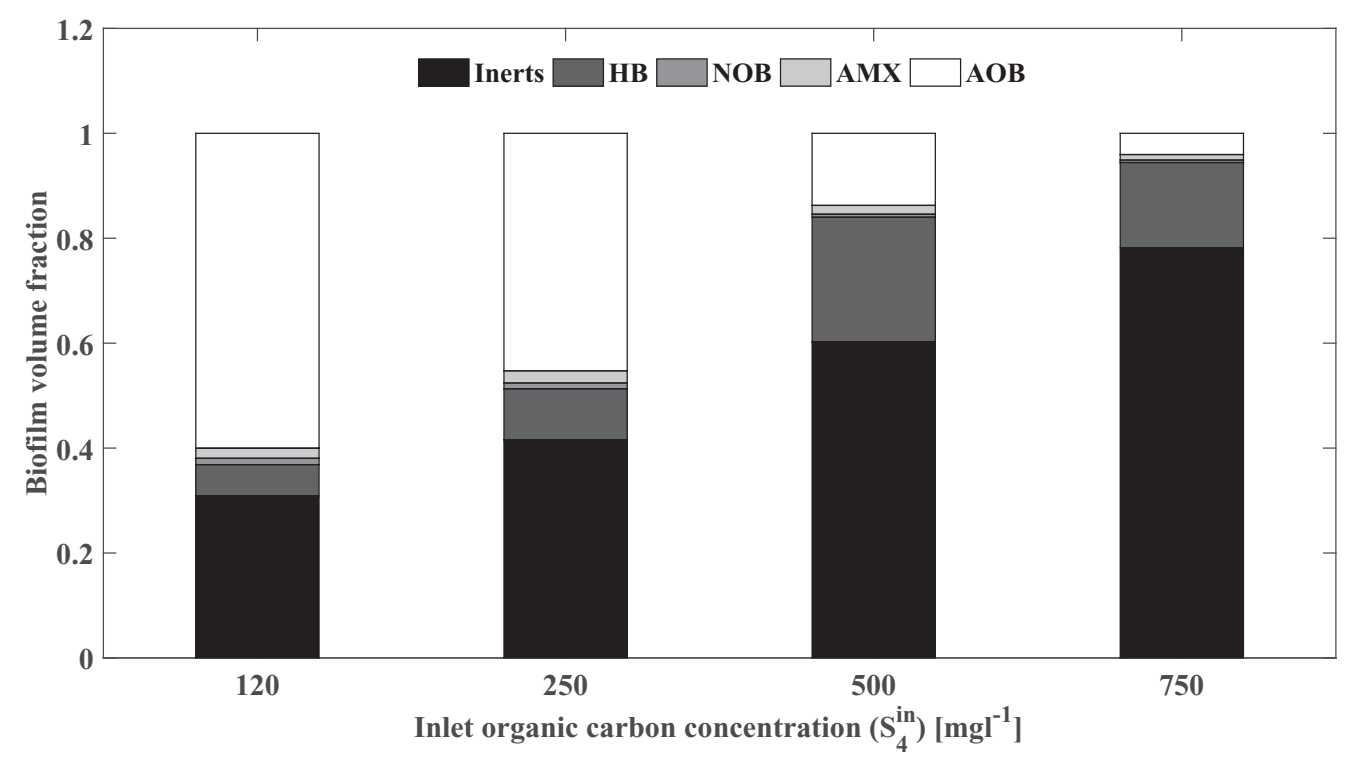

FIGURE 6. Total biofilm volume fractions at different inlet organic carbon concentrations $\left(S_{4}^{\text {in }}\right)$ after 50 days simulation time.

$$
\begin{gathered}
r_{4}=k_{\mathrm{col}, 4} \frac{\Psi_{4}}{k_{\varphi, 4}+\Psi_{4}}\left(\frac{S_{4}}{K_{4,4}+S_{4}} \frac{S_{5}}{K_{4,5}+S_{5}} \frac{S_{1}}{S_{1}+K_{4,1}}+\beta_{1} \frac{K_{4,5}}{K_{4,5}+S_{5}} \frac{S_{4}}{K_{4,4}+S_{4}} \frac{S_{3}}{K_{4,3}+S_{3}} \frac{S_{3}}{S_{2}+S_{3}}\right. \\
\left.\times \frac{S_{1}}{S_{1}+K_{4,1}}+\beta_{2} \frac{K_{4,5}}{K_{4,5}+S_{5}} \frac{S_{4}}{K_{4,4}+S_{4}} \frac{S_{2}}{K_{4,2}+S_{2}} \frac{S_{2}}{S_{2}+S_{3}} \frac{S_{1}}{S_{1}+K_{4,1}}\right) .
\end{gathered}
$$

The growth rate terms $r_{2}$ and $r_{4}$ for $X_{2}$ (AMX) and $X_{4}(\mathrm{HB})$, respectively, indicate that the transition of bacteria from planktonic state $\Psi_{2}, \Psi_{4}$ into the sessile state $X_{2}, X_{4}$ is controlled by the formation of specific environmental niches connected to the local concentration of dissolved substrates. As in Model 1, consider the second and fourth equations in (5.1) with $r_{M, 2}$ and $r_{M, 4}$ given by (5.5) and (5.7), respectively. If it is supposed that $r_{2}=r_{4}=0$, then the mentioned equations with initial condition $f_{2}(z, 0)=f_{4}(z, 0)=0$ admit the unique solution $f_{2}(z, t)=f_{4}(z, t)=0$ and the species $X_{2}$ and $X_{4}$ cannot develop.

The initial boundary conditions for $S_{j}$ and the net substrate conversion rates are the same as Model 1, formulas (5.12), (5.13) and (5.14)-(5.18), respectively. The initial conditions for $S_{j}^{*}$ are given by (5.20). The initial boundary conditions for $\Psi_{i}$ are same as Model 1, formula (5.23). The initial conditions for $\psi_{i}^{*}$ are the following:

$$
\psi_{1}^{\text {in }}=0, \psi_{2}^{\text {in }}=1.0 \mathrm{mg} \mathrm{COD} / \mathrm{L}, \psi_{3}^{\text {in }}=0, \psi_{4}^{\text {in }}=1.2 \mathrm{mg} \mathrm{COD} / \mathrm{L}, \psi_{5}^{\text {in }}=0 .
$$

Note that, by using the same arguments as Model 1, it can be shown that $\Psi_{1}(z, t)=0$, $\psi_{1}^{*}(t)=0, \Psi_{3}(z, t)=0, \psi_{3}^{*}(t)=0, \Psi_{5}(z, t)=0, \psi_{5}^{*}(t)=0$.

The operational parameters of the biofilm reactor are the same as Model 1.

In Figure 9, the simulation results for the multispecies biofilm system with two invading species are reported. Differently from Model 1, the $X_{4}(\mathrm{HB})$ invasion is very fast and 


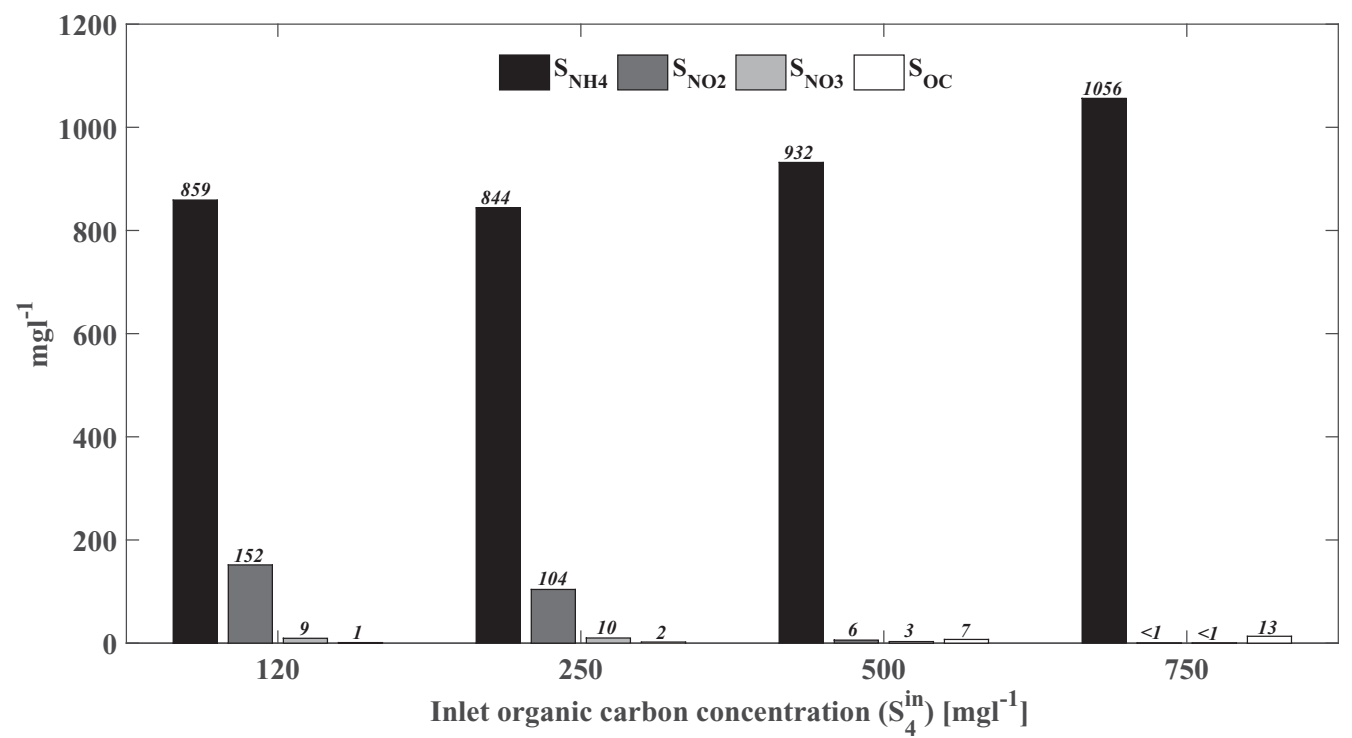

FIGURE 7. Substrate concentrations within the bulk liquid at different inlet organic carbon concentrations $\left(S_{4}^{\text {in }}\right)$ after 50 days simulation time. The values reported have been rounded to the nearest integer.

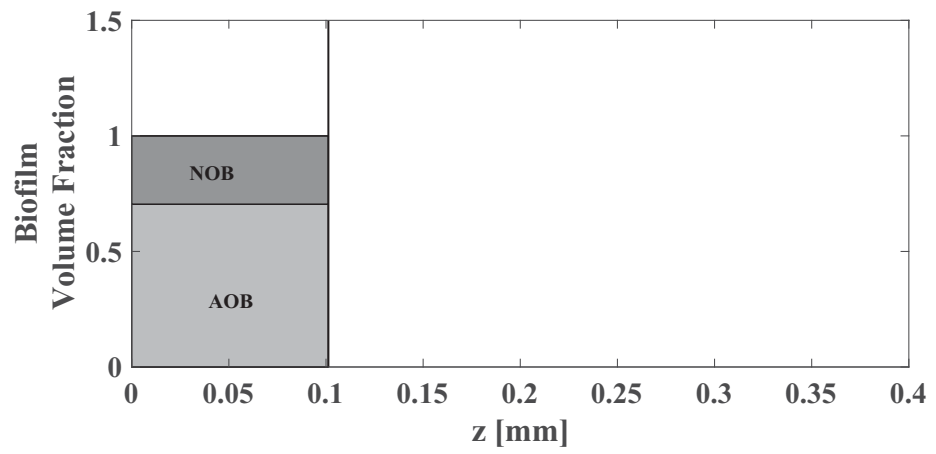

FIGURE 8. Initial biofilm configuration for Model 2. The substratum is placed at $z=0$.

it is already visible after 5 days of simulation time (Figure 9(B)). This is due to the different environmental conditions that trigger the invasion of the two microbial species. Indeed the establishment of $X_{2}(\mathrm{AMX})$ is dependent on the formation of an anoxic zone within the biofilm, while $X_{4}(\mathrm{HB})$ are facultative bacteria and can grow in both aerobic and anoxic environments. After 20 days, the biofilm configuration is the same of the previous application (Figures 3(A) and 9(C)) and as we can expect, the further evolution of the system is practically the same for the two cases studied (Figures 3(B) and 9(D)). Simulation results confirm model capability of predicting the invasion phenomenon on time and space. Indeed, the model is able to predict the delays between the $X_{2}$ (AMX) and $X_{4}(\mathrm{HB})$ colonizations and the location where the two planktonic species establish. To the best of our knowledge, such results cannot be achieved by the existing continuum biofilm 
(A)

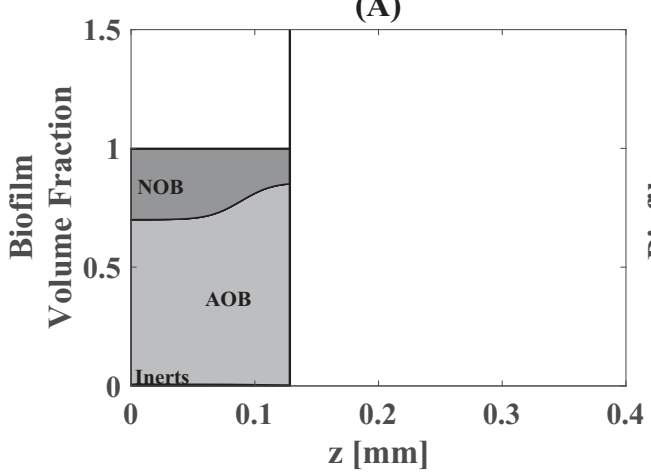

(C)

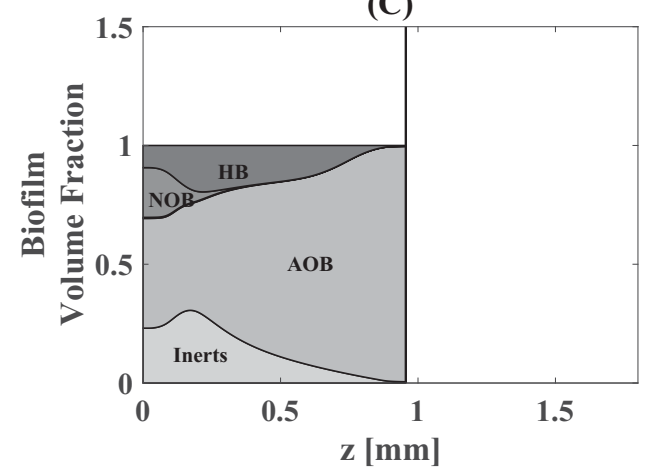

(B)

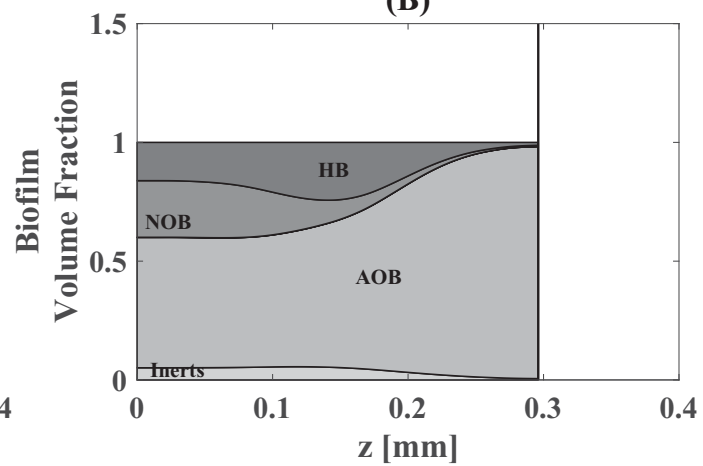

(D)

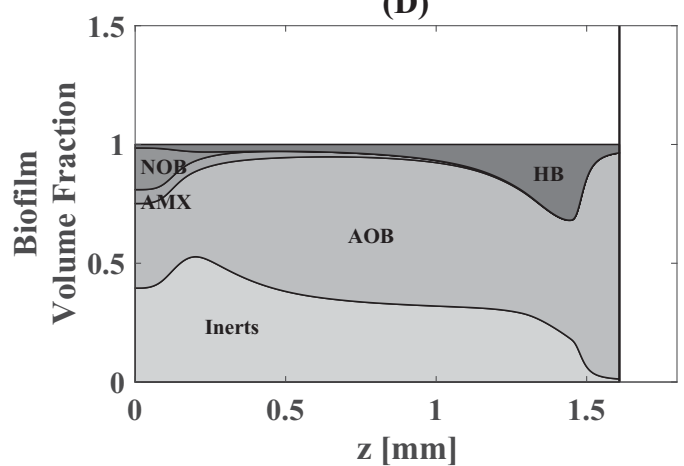

FIGURE 9. Microbial species distribution of a multispecies biofilm undergoing $\Psi_{2}$ and $\Psi_{4}$ colonization after 2(A), 5(B), 20(C), 50(D) days simulation time. The substratum is placed at $z=0$.

models but they might have a significant impact on the development of new strategies for such biofilm reactors operation.

\section{Conclusion}

In this work, the qualitative analysis of the free boundary problem related to the invasion phenomenon in biofilm reactors has been discussed. The model takes into account the dynamics of sessile species, nutrients and microbial products and planktonic cells. The latter diffuse from the bulk liquid within the biofilm matrix, where they might switch their status from motile to sessile and thus colonize the pre-existing biofilm. The dynamics of the bulk liquid have been explicitly modelled by considering two systems of nonlinear ordinary differential equations which derive from mass conservation principles. An existence and uniqueness result has been provided for the related free boundary value problem by using the method of characteristics and the fixed point theorem. It is important to notice that the planktonic species are just provided by the bulk liquid; however, the reverse process which accounts for the switch from the sessile to the planktonic form of life might occur under specific conditions. This phenomenon could be explicitly taken into account by considering a direct dependence of the free planktonic cell reaction rates on the concentration of the sessile bacteria. The same methodology adopted in this work 
could be easily adapted to address the existence and uniqueness questions of this new system. In addition, future work, based on a more solid experimental evidence, could be focused on a more detailed description of the free cells movement within the biofilm, which might imply the consideration of $D_{M, i}$ as a function of the biofilm composition. Numerical simulations related to a real biofilm system have been performed. Two specific model applications have been analysed. Simulation results demonstrate the underlying conclusion that the invasion model can be effectively used as a predictive tool to develop specific reactor operation strategies. More precisely, the model can be used to predict the optimal operational conditions (dilution rate, oxygen concentration, external carbon content addition, etc.) which favour the establishment of the desired microbial syntrophy or viceversa, when all the operational conditions are fixed, to verify the performance of the biological system. Further developments might be related to the definition of a calibration protocol through experimental data as well as the generalization to problems regarding more complex biological cases and the consideration of stability questions $[26,27]$.

\section{Acknowledgements}

L Frunzo and MR Mattei acknowledge the Progetto Giovani G.N.F.M. 2017 Analisi di Sistemi Biologici Complessi.

\section{References}

[1] Boltz, J. P., Smets, B. F., Rittmann, B. E., van Loosdrecht, M. C., Morgenroth, E. \& Daigger, G. T. (2017) From biofilm ecology to reactors: A focused review. Water Sci. Technol. 75(8), 1753-1760.

[2] Mattei, M. R., Frunzo, L., D’Acunto, B., Esposito, G. \& Pirozzi, F. (2015) Modelling microbial population dynamics in multispecies biofilms including anammox bacteria. Ecol. Modell. 304, 44-58.

[3] Ward, J. P. \& King, J. R. (2012) Thin-film modelling of biofilm growth and quorum sensing. J. Eng. Math. 73(1), 71-92.

[4] Houry, A., Gohar, M., Deschamps, J., Tischenko, E., Aymerich, S., Gruss, A., \& Briandet, R. (2012) Bacterial swimmers that infiltrate and take over the biofilm matrix. PNAS 109(32), 13088-13093.

[5] Eberl, H. J., Parker, D. F., \& Van Loosdrecht, M. (2001) A new deterministic spatiotemporal continuum model for biofilm development. Comput. Math. Methods Med. 3(3), 161-175.

[6] Sonner, S., Efendiev, M. A. \& Eberl, H. J. (2015) On the well-posedness of mathematical models for multicomponent biofilms. Math. Methods Appl. Sci. 38(17), 3753-3775.

[7] Zhang, T., Cogan, N. G. \& Wang, Q. (2008) Phase field models for biofilms. I. Theory and one-dimensional simulations. SIAM J. Appl. Math. 69(3), 641-669.

[8] Picioreanu, C., Kreft, J. U. \& Van Loosdrecht, M. C. (2004) Particle-based multidimensional multispecies biofilm model. Appl. Environ. Microbiol. 70(5), 3024-3040.

[9] Jayathilake, P. G., Gupta, P., Li, B., Madsen, C., Oyebamiji, O., González-Cabaleiro, R., Rushton, S., Bridgens, B., Swailes, D., Allen, B., McGough, A. S., Zuliani, P., Ofiteru, I. D., Wilkinson, D., Chen, J., \& Curtis, T. (2017) A mechanistic individual-based model of microbial communities. Plos one 12(8), e0181965. 
[10] Mattei, M. R., Frunzo, L., D’Acunto, B., Pechaud, Y., Pirozzi, F. \& Esposito, G. (2018) Continuum and discrete approach in modeling biofilm development and structure: A review. J. Math. Biol. 76(4), 945-1003.

[11] Wanner, O. \& Gujer, W. (1986) A multispecies biofilm model. Biotechnol. Bioeng. 28(3), 314-328.

[12] Klapper, I. \& Szomolay, B. (2011 ) An exclusion principle and the importance of mobility for a class of biofilm models. Bull. Math. Biol. 73(9), 2213-2230.

[13] Wanner, O. \& Reichert, P. (1996) Mathematical modeling of mixed-culture biofilms. Biotechnol. Bioeng. 49(2), 172-184.

[14] D’Acunto, B., Frunzo, L., Klapper, I. \& Mattei, M. (2015) Modeling multispecies biofilms including new bacterial species invasion. Math. Biosci. 259, 20-26.

[15] MAšíc, A. \& EberL, H. J. (2014) A modeling and simulation study of the role of suspended microbial populations in nitrification in a biofilm reactor. Bull. Math. Biol. 76(1), 27-58.

[16] Szomolay, B. (2008) Analysis of a moving boundary value problem arising in biofilm modelling. Math. Methods Appl. Sci. 31(15), 1835-1859.

[17] Szomolay, B., Klapper, I. \& Dindos, M. (2010) Analysis of adaptive response to dosing protocols for biofilm control. SIAM J. Appl. Math. 70(8), 3175-3202.

[18] D’Acunto, B., Frunzo, L. \& Mattei, M. R. (2016) Qualitative analysis of the moving boundary problem for a biofilm reactor model. J. Math. Anal. Appl. 438(1), 474-491.

[19] Frunzo, L. \& Mattei, M. R. (2017) Qualitative analysis of the invasion free boundary problem in biofilms. Ricerche di Matematica 66(1), 171-188.

[20] Dockery, J. \& Klapper, I. (2002) Finger formation in biofilm layers. SIAM J. Appl. Math. 62(3), 853-869.

[21] Rogers, S. S., Van Der Walle, C. \& Waigh, T. A. (2008) Microrheology of bacterial biofilms in vitro: Staphylococcus aureus and Pseudomonas aeruginosa. Langmuir 24(23), 13549-13555.

[22] D’Acunto, B., Esposito, G., Frunzo, L. \& Pirozzi, F. (2011) Dynamic modeling of sulfate reducing biofilms. Comput. Math. Appl. 62(6), 2601-2608.

[23] Cao, Y., van Loosdrecht, M. C. \& Daigger, G. T. (2017) Mainstream partial nitritationanammox in municipal wastewater treatment: status, bottlenecks, and further studies. Appl. Microbiol. Biotechnol. 101(4), 1365-1383.

[24] Abbas, F., Sudarsan, R. \& Eberl, H. J. (2011) Longtime behavior of one-dimensional biofilm models with shear dependent detachment rates. Math. Biosci. Eng. 9(2), 215-239.

[25] Ostace, G. S., Cristea, V. M. \& AGachi, P. (2011) Cost reduction of the wastewater treatment plant operation by MPC based on modified ASM1 with two-step nitrification/denitrification model. Comput. Chem. Eng. 35(11), 2469-2479.

[26] Capone, F. \& De Luca, R. 2012 Onset of convection for ternary fluid mixtures saturating horizontal porous layers with large pores. Rend. Lincei Mat. Appl. 23(4), 405-428.

[27] Capone, F., De Cataldis, V., De Luca, R. \& Torcicollo, I. (2014) On the stability of vertical constant throughflows for binary mixtures in porous layers. Int. J. Non-Linear Mech. 59, 1-8.

\section{Appendix A}

Proof of Theorem 1. Consider the map $\mathbf{y}=A \mathbf{x}^{*}$, where $A\left(\mathbf{x}^{*}\right)$ designates the right-hand side of equations (4.1)-(4.7). Denote by $\mathbf{V}$ the vector space of the continuous functions $x_{h}^{*}, h=1, \ldots, 3 n+2+2 m$, on $I=\left[0, L_{0}\right] \times[0, T]$. Let us first prove that A maps V into itself.

Let $K_{n+2+m+j}=K_{n+2+j}+3 M_{n+2+m+j} L_{0}$ and $K_{2 n+2+2 m+i}=K_{n+2+2 m+i}$ $+3 M_{2 n+2+2 m+i} L_{0}$. First, hypothesis (iv), jointly with $x_{n+2}^{*} \geqslant 1$, implies $F_{n+2} \geqslant 0$. Then,

$$
\begin{gathered}
\left|y_{i}-\varphi_{i}\right| \leqslant M_{i} T \leqslant K_{i}, i=1, \ldots, n, \\
\left|y_{n+1}-z_{0}\right| \leqslant M_{n+1} T L_{0} \leqslant K_{n+1},
\end{gathered}
$$




$$
\begin{gathered}
1 \leqslant y_{n+2} \leqslant 1+M_{n+2} T \leqslant 1+K_{n+2}, \\
\left|y_{n+2+j}-S_{j}^{\mathrm{in}}\right| \leqslant M_{n+2+j} T L_{0} \leqslant K_{n+2+j}, j=1, \ldots, m, \\
\left|y_{n+2+m+j}-S_{j}^{\mathrm{in}}\right| \leqslant\left|y_{n+2+j}-S_{j}^{\mathrm{in}}\right|+3 M_{n+2+m+j} L_{0} \leqslant K_{n+2+m+j}, j=1, \ldots, m, \\
\left|y_{n+2+2 m+i}-\psi_{i}^{\mathrm{in}}\right| \leqslant M_{n+2+2 m+i} T L_{0} \leqslant K_{n+2+2 m+i}, i=1, \ldots, n, \\
\left|y_{2 n+2+2 m+i}-\psi_{i}^{\mathrm{in}}\right| \leqslant\left|y_{n+2+2 m+i}-\psi_{i}^{\mathrm{in}}\right|+3 M_{2 n+2+2 m+i} L_{0} \\
\leqslant K_{2 n+2+2 m+i}, i=1, \ldots, n,
\end{gathered}
$$

which is the desired result.

Let us now prove that $\mathrm{A}$ is a contractive map. Assume $F_{h}$ Lipschitz continuous functions with respect to $x_{h}^{*}, h=1, \ldots, 3 n+2+2 m$

$$
\begin{gathered}
\left|F_{i}\left(\tau, \mathbf{x}^{*}\right)-F_{i}\left(\tau, \tilde{\mathbf{x}}^{*}\right)\right| \leqslant \lambda_{i} \sum_{h=1}^{3 n+2+2 m}\left|x_{h}^{*}-\tilde{x}_{h}^{*}\right|, i=1, \ldots, n+2+m, \\
\left|F_{i}^{1}\left(\tau, \mathbf{x}^{*}\right)-F_{i}^{1}\left(\tau, \tilde{\mathbf{x}}^{*}\right)\right| \leqslant \lambda_{i}^{1} \sum_{h=1}^{3 n+2+2 m}\left|x_{h}^{*}-\tilde{x}_{h}^{*}\right|, i=n+m+3, \ldots, n+2+2 m, \\
\left|F_{i}^{2}\left(\tau, \mathbf{x}^{*}\right)-F_{i}^{2}\left(\tau, \tilde{\mathbf{x}}^{*}\right)\right| \leqslant \lambda_{i}^{2} \sum_{h=1}^{3 n+2+2 m}\left|x_{h}^{*}-\tilde{x}_{h}^{*}\right|, i=n+m+3, \ldots, n+2+2 m, \\
\left|F_{i}^{3}\left(\tau, \mathbf{x}^{*}\right)-F_{i}^{3}\left(\tau, \tilde{\mathbf{x}}^{*}\right)\right| \leqslant \lambda_{i}^{3} \sum_{h=1}^{3 n+2+2 m}\left|x_{h}^{*}-\tilde{x}_{h}^{*}\right|, i=n+m+3, \ldots, n+2+2 m, \\
\left|F_{i}\left(\tau, \mathbf{x}^{*}\right)-F_{i}\left(\tau, \tilde{\mathbf{x}}^{*}\right)\right| \leqslant \lambda_{i} \sum_{h=1}^{3 n+2+2 m}\left|x_{h}^{*}-\tilde{x}_{h}^{*}\right|, i=n+3+2 m, \ldots, 2 n+2+2 m, \\
\left|F_{i}^{1}\left(\tau, \mathbf{x}^{*}\right)-F_{i}^{1}\left(\tau, \tilde{\mathbf{x}}^{*}\right)\right| \leqslant \lambda_{i}^{1} \sum_{h=1}^{3 n+2+2 m}\left|x_{h}^{*}-\tilde{x}_{h}^{*}\right|, i=2 n+2 m+3, \ldots, 3 n+2+2 m, \\
\left|F_{i}^{3}\left(\tau, \mathbf{x}^{*}\right)-F_{i}^{3}\left(\tau, \tilde{\mathbf{x}}^{*}\right)\right| \leqslant \lambda_{i}^{3} \sum_{h=1}^{3 n+2+2 m}\left|x_{h}^{*}-\tilde{x}_{h}^{*}\right|, i=2 n+2 m+3, \ldots, 3 n+2+2 m . \\
F_{i}^{2}\left(\tau, \mathbf{x}^{*}\right)-F_{i}^{2}\left(\tau, \tilde{\mathbf{x}}^{*}\right)\left|\leqslant \lambda_{i}^{2} \sum_{h=1}^{3 n+2+2 m}\right| x_{h}^{*}-\tilde{x}_{h}^{*} \mid, i=2 n+2 m+3, \ldots, 3 n+2+2 m .
\end{gathered}
$$

and introduce the norm

$$
\left\|\mathbf{x}^{*}\right\|=\sum_{h=1}^{3 n+2 m+2} \max _{I} \exp (-\gamma t)\left|x_{h}^{*}\right|
$$

with $\gamma$ a positive constant. 
Consider $\tilde{x}^{*} \in \mathbf{V}$ with $\tilde{y}=A \tilde{x}^{*}$. It follows

$$
\begin{gathered}
\left|y_{i}-\tilde{y}_{i}\right| \exp (-\gamma t) \leqslant\left(\lambda_{i} / \gamma\right)|| \mathbf{x}^{*}-\tilde{\mathbf{x}}^{*} \|, i=1, \ldots, n, \\
\left|y_{n+1}-\tilde{y}_{n+1}\right| \exp (-\gamma t) \leqslant\left(\lambda_{n+1} L_{0} / \gamma\right)\left\|\mathbf{x}^{*}-\tilde{\mathbf{x}}^{*}\right\|, \\
\left|y_{n+2}-\tilde{y}_{n+2}\right| \exp (-\gamma t) \leqslant\left(\lambda_{n+2} / \gamma\right)\left\|\mathbf{x}^{*}-\tilde{\mathbf{x}}^{*}\right\|, \\
\left|y_{n+2+j}-\tilde{y}_{n+2+j}\right| \exp (-\gamma t) \leqslant\left(\lambda_{n+2+j} L_{0} / \gamma\right)|| \mathbf{x}^{*}-\tilde{\mathbf{x}}^{*} \|, j=1, \ldots, m, \\
\left|y_{n+2+m+j}-\tilde{y}_{n+2+m+j}\right| \exp (-\gamma t) \leqslant\left(\lambda_{n+2+m+j}^{1}+\lambda_{n+2+m+j}^{2}\right. \\
\left.+\lambda_{n+2+m+j}^{3}\right) L_{0}|| \mathbf{x}^{*}-\tilde{\mathbf{x}}^{*} \|, j=1, \ldots, m, \\
\left|y_{n+2+2 m+i}-\tilde{y}_{n+2+2 m+i}\right| \exp (-\gamma t) \leqslant\left(\lambda_{n+2+2 m+i} L_{0} / \gamma\right)\left\|\mathbf{x}^{*}-\tilde{\mathbf{x}}^{*}\right\|, i=1, \ldots, n, \\
\left|y_{2 n+2+2 m+i}-\tilde{y}_{2 n+2+2 m+i}\right| \exp (-\gamma t) \leqslant\left(\lambda_{2 n+2+2 m+i}^{1}+\lambda_{2 n+2+2 m+i}^{2}\right. \\
\left.+\lambda_{2 n+2+2 m+i}^{3}\right) L_{0}\left\|\mathbf{x}^{*}-\tilde{\mathbf{x}}^{*}\right\|, i=1, \ldots, n .
\end{gathered}
$$

Hence,

$$
\left\|\mathbf{y}^{*}-\tilde{\mathbf{y}}^{*}\right\| \leqslant \Lambda|| \mathbf{x}^{*}-\tilde{\mathbf{x}}^{*} \|
$$

where

$$
\begin{gathered}
\Lambda=\Lambda_{1}+\Lambda_{2}, \\
\Lambda_{1}=\frac{1}{\gamma}\left(\sum_{i=1}^{n} \lambda_{i}+\lambda_{n+1} L_{0}+\lambda_{n+2}+\sum_{j=1}^{m} \lambda_{n+2+j}+\sum_{i=1}^{n} \lambda_{n+2+2 m+i}\right), \\
\Lambda_{2}=L_{0}\left(\sum_{j=1}^{m}\left(\lambda_{n+2+m+j}^{1}+\lambda_{n+2+m+j}^{2}+\lambda_{n+2+m+j}^{3}\right)+\sum_{i=1}^{n}\left(\lambda_{2 n+2+2 m+i}^{1}\right.\right. \\
\left.\left.+\lambda_{2 n+2+2 m+i}^{2}+\lambda_{2 n+2+2 m+i}^{3}\right)\right) .
\end{gathered}
$$

Selecting $\gamma$ such that $\Lambda_{1}<\epsilon, \forall \epsilon>0$ and $L_{0}$ small enough such that

$$
\begin{gathered}
L_{0} \leqslant(1-\epsilon)\left(\sum_{j=1}^{m}\left(\lambda_{n+2+m+j}^{1}+\lambda_{n+2+m+j}^{2}+\lambda_{n+2+m+j}^{3}\right)+\sum_{i=1}^{n}\left(\lambda_{2 n+2+2 m+i}^{1}\right.\right. \\
\left.\left.+\lambda_{2 n+2+2 m+i}^{2}+\lambda_{2 n+2+2 m+i}^{3}\right)\right)^{-1},
\end{gathered}
$$

then $\Lambda<1$ and the theorem is proved. 
Appendix B

Table B1. Petersen matrix of the model application

\begin{tabular}{|c|c|c|c|c|c|c|c|c|c|c|c|c|}
\hline & Components & 1 & 2 & 3 & 4 & 5 & 6 & 7 & 8 & 9 & 10 & \\
\hline & Process & $S_{1}$ & $S_{2}$ & $S_{3}$ & $S_{4}$ & $S_{5}$ & $X_{1}$ & $X_{2}$ & $X_{3}$ & $X_{4}$ & $X_{5}$ & Process rate \\
\hline 1 & Growth of $X_{1}$ & $-\frac{1}{Y_{1}}-i_{N, B}$ & $\frac{1}{Y_{1}}$ & & & $1-\frac{3.43}{Y_{1}}$ & 1 & & & & & $\mu_{1} X_{1}$ \\
\hline 2 & Growth of $X_{2}$ & $-\frac{1}{Y_{2}}-i_{N, B}$ & $\left(\frac{1}{Y_{2}}+\frac{1}{1.14}\right)$ & $\frac{1}{1.14}$ & & & & 1 & & & & $\mu_{2} X_{2}$ \\
\hline 3 & Growth of $X_{3}$ & ${ }^{r_{2}}-i_{N, B}$ & ${ }^{Y_{2}}-\frac{1}{Y_{3}}$ & $\begin{array}{l}1.14 \\
\frac{1}{Y_{3}}\end{array}$ & & $1-\frac{1.14}{Y_{3}}$ & & & 1 & & & $\mu_{3} X_{3}$ \\
\hline 4 & Aerobic growth of $X_{4}$ & $-i_{N, B}$ & & & $-\frac{1}{Y_{4}}$ & $1-\frac{1}{Y_{4}}$ & & & & 1 & & $\mu_{4,1} X_{4}$ \\
\hline 5 & Growth of $X_{4}$ on $S_{3}$ & $-i_{N, B}$ & $-\left(1-\frac{1}{Y_{4}}\right) \frac{1}{1.14}$ & $\left(1-\frac{1}{Y_{4}}\right) \frac{1}{1.14}$ & $-\frac{1}{Y_{4}}$ & & & & & 1 & & $\mu_{4,2} X_{4}$ \\
\hline 6 & Growth of $X_{4}$ on $S_{2}$ & $-i_{N, B}$ & $\left(1-\frac{1}{Y_{4}}\right) \frac{1}{1.72}$ & & $-\frac{1}{Y_{4}}$ & & & & & 1 & & $\mu_{4,3} X_{4}$ \\
\hline 7 & Decay of $X_{1}$ & & & & & & -1 & & & & +1 & $k_{d, 1} X_{1}$ \\
\hline 8 & Decay of $X_{2}$ & & & & & & & -1 & & & +1 & $k_{d, 2} X_{2}$ \\
\hline 9 & Decay of $X_{3}$ & & & & & & & & -1 & & +1 & $k_{d, 3} X_{3}$ \\
\hline \multirow[t]{2}{*}{10} & Decay of $X_{4}$ & & & & & & & & & -1 & +1 & $k_{d, 4} X_{4}$ \\
\hline & & $\tilde{z}^{a}$ & $\underbrace{n}_{0}$ & $\tilde{b}_{0}^{n}$ & $\ddot{\vdots}$ & $\check{D}$ & $\underset{\varpi}{0}$ & $\frac{3}{x}$ & $\begin{array}{l}\text { Ż } \\
\text { O }\end{array}$ & $\boldsymbol{\Xi}$ & $\frac{\Xi}{\bar{m}}$ & \\
\hline
\end{tabular}


Table B2. Kinetic rate expressions for model application

Process

Rate expression

1. Growth of $X_{1}$

2. Growth of $X_{2}$

3. Growth of $X_{3}$

4. Aerobic growth of $X_{4}$

5. Growth of $X_{4}$ on $S_{3}$

6. Growth of $X_{4}$ on $S_{2}$

7. Decay of $X_{1}$

8. Decay of $X_{2}$

9. Decay of $X_{3}$

10. Decay of $X_{4}$

$\mu_{1} X_{1}=\mu_{\max , 1} \frac{S_{1}}{K_{1,1}+S_{1}} \frac{S_{5}}{K_{1,5}+S_{5}} X_{1}$
$\mu_{2} X_{2}=\mu_{\max , 2} \frac{K_{2,5}}{K_{2,5}+S_{5}} \frac{S_{1}}{K_{2,1}+S_{1}} \frac{S_{2}}{K_{2,2}+S_{2}} X_{2}$
$\mu_{3} X_{3}=\mu_{\max , 3} \frac{S_{2}}{K_{3,2}+S_{2}} \frac{S_{5}}{K_{3,5}+S_{5}} \frac{S_{1}}{S_{1}+K_{3,1}} X_{3}$
$\mu_{4,1} X_{4}=\mu_{\max , 4} \frac{S_{4}}{K_{4,4}+S_{4}} \frac{S_{5}}{K_{4,5}+S_{5}} \frac{S_{1}}{S_{1}+K_{4,1}} X_{4}$
$\mu_{4,2} X_{4}=\beta_{1} \mu_{\max , 4} \frac{K_{4,5}}{K_{4,5}+S_{5}} \frac{S_{4}}{K_{4,4}+S_{4}} \frac{S_{3}}{K_{4,3}+S_{3}} \frac{S_{3}}{S_{3}+S_{2}} \frac{S_{1}}{S_{1}+K_{4,1}} X_{4}$
$\mu_{4,3} X_{4}=\beta_{2} \mu_{\max , 4} \frac{K_{4,5}}{K_{4,5}+S_{5}} \frac{S_{4}}{K_{4,4}+S_{4}} \frac{S_{2}}{K_{4,2}+S_{2}} \frac{S_{2}}{S_{3}+S_{2}} \frac{S_{1}}{S_{1}+K_{4,1}} X_{4}$
$k_{d, 1} X_{1}$
$k_{d, 2} X_{2}$
$k_{d, 3} X_{3}$
$k_{d, 4} X_{4}$

